\title{
EXTREMAL FUNCTIONS FOR MODULES OF SYSTEMS OF MEASURES
}

\author{
MELKANA BRAKALOVA ${ }^{\dagger}$, IRINA MARKINA ${ }^{\ddagger}$, AND ALEXANDER VASIL’EV $^{\ddagger}$
}

\begin{abstract}
We study Fuglede's p-module of systems of measures in condensers in Euclidean spaces and on polarizable Carnot groups. We apply and generalize a result by Rodin, which provides an explicit method for finding the extremal function and the 2-module of a foliated family of curves in $\mathbb{R}^{2}$, to a variety of settings. In the planar case, we apply Rodin's method to obtain estimates for the conformal module of a parallelogram and of a ring domain using directional dilatations. In $\mathbb{R}^{n}$, we identify the extremal function and compute the $p$-module of images of families of connecting curves and of separating sets with respect to the plates of a condenser under homeomorphisms of certain regularity. Then we calculate the module and find the extremal measures for the spherical ring domain on polarizable Carnot groups and extend Rodin's theorem to the spherical ring domain on the Heisenberg group.
\end{abstract}

\section{Contents}

1. Introduction 2

2. Rodin's theorem and extremality in $\mathbb{R}^{2}$

2.1. Extremal functions and extremal families of curves 5

2.2. Rodin's theorem and applications 7

3. Rodin's theorem in Euclidean spaces 13

3.1. Module of a system of measures in Euclidean spaces 13

3.2. Rodin's theorem for families of connecting curves in a condenser 15

3.3. Modules of families of separating sets in a condenser 16

3.4. Proof of Theorem 3 and 4 - 20

4. Extremal measures on polarizable groups 25

2010 Mathematics Subject Classification. Primary: 30C65, 30C85; Secondary: 26B10, $46 \mathrm{E} 35$.

Key words and phrases. Module of a family of curves, extremal metric, Sobolev space, nilpotent Lie group, Carnot group.

The author ${ }^{\dagger}$ was partially supported by a Faculty Research Grant, Fordham University, USA, and by the Norwegian Research Council grant \#204726/V30. The authors ${ }^{\ddagger}$ were partially supported by the grants of the Norwegian Research Council \#204726/V30 and \#213440/BG, as well as by EU FP7 IRSES program STREVCOMS, grant no. PIRSESGA-2013-612669. 
4.1. Definition of polarizable groups 25

4.2. The $p$-module of $\Gamma\left(R_{a b} ; S_{a}, S_{b}\right)$ on polarizable groups 29

4.3. The $p$-module of a family of separating sets in $R_{a b}$

4.4. Admissibility of $\rho_{0}$ for $M_{q}(E) \quad 37$

4.5. Twisting map of the spherical ring in the Heisenberg group 41

References 45

\section{INTRODUCTION}

Let $\Omega$ be a bounded domain in a polarizable Carnot group $G$, a particular case of which is the Euclidean space $\mathbb{R}^{n}, n \geq 2$, and let $D_{0}$ and $D_{1}$ be two disjoint compacts in the closure $\bar{\Omega}$ of $\Omega$. The triple $\left(\Omega ; D_{0}, D_{1}\right)$ is called a condenser in $G$, and the compacts $D_{0}$ and $D_{1}$ - its plates. Two important quantities related to the condenser $\left(\Omega ; D_{0}, D_{1}\right)$ are the module of the family of curves $\Gamma\left(\Omega ; D_{0}, D_{1}\right)$ connecting the compacts $D_{0}$ and $D_{1}$ in $\Omega$, and the module of the surfaces, or more generally, the sets $\Sigma\left(\Omega ; D_{0}, D_{1}\right)$ separating $D_{0}$ and $D_{1}$ in $\Omega$. Observe, that the module of $\Gamma\left(\Omega ; D_{0}, D_{1}\right)$ is closely related to the notion of capacity of $D_{0}$ and $D_{1}$ relatively to $\Omega$, see e.g., [6, 29, 39, 54, 74, 75, 89, 90. Since the notion of Lipschitz surfaces is somewhat restrictive for the development of the theory of modules and related topics, it is convenient to use the notion of modules of systems of measures introduced by Fuglede [25] in 1957.

We first recall the definition. Let $(X, \mathfrak{M}, m)$ be an abstract measure space with a fixed measure $m: \mathfrak{M} \rightarrow[0,+\infty]$ defined on the $\sigma$-algebra $\mathfrak{M}$ of subsets in $X$. We denote by $\mathcal{M}$ the system of all measures $\mu$ in $X$, whose domains of definition contain $\mathfrak{M}$. With an arbitrary system of measures $E \subset \mathcal{M}$ we associate the class of non-negative measurable functions $\rho$ defined in $X$ and satisfying the condition

$$
\int_{X} \rho d \mu \geqslant 1, \quad \mu \in E .
$$

We call such $\rho$ admissible, and we write $\rho \wedge \mu$ if (1) holds for a measure $\mu$, and $\rho \wedge E$ if (1) holds for every $\mu \in E$.

Definition 1. For $0<p<\infty$, the p-module $M_{p}(E)$ of the system of measures $E$ is defined as

$$
M_{p}(E)=\inf _{\rho \wedge E} \int_{X} \rho^{p} d m
$$

interpreted as $+\infty$ if the set $\{\rho: \rho \wedge E\}$ is empty. A function $\rho_{0}, \rho_{0} \wedge E$, is called extremal for the p-module $M_{p}(E)$ if $M_{p}(E)=\int_{X} \rho_{0}^{p} d m$. 
Definition 1 is a natural generalization of the concept of the module of a family of curves in $\mathbb{R}^{n}, n \geq 2$. Given a family $\Gamma$ of locally rectifiable curves in space $X=\mathbb{R}^{n}$, one can regard $\mathfrak{M}$ as the Borel $\sigma$-algebra, $m$ as the Lebesgue $n$-dimensional measure, and $\mu$ as the arc-length of a curve which is a measure associated with this curve. This construction for the case of $\mathbb{R}^{n}$ was carefully developed in detail in [61, Chapter 2] and also in [25].

We call a system of measures $E_{0} \subseteq E$ extremal for $M_{p}(E)$ if

$$
\int_{X} \rho_{0} d \mu=1 \quad \text { and } \quad M_{p}(E)=M_{p}\left(E_{0}\right)=\int_{X} \rho_{0} d \mu
$$

for $M_{p}$-almost all $\mu \in E_{0}$. If, in addition, $E_{0}=E$ for $M_{p}$-almost all $\mu$, we call $E_{0}$ a complete extremal system of measures. The family of extremal measures does not always exist and is not, in general, uniquely determined, although it is in the cases we consider. We consider this in more detail in the special case of extremal family of curves in Section 2.

The above definition of an extremal function and measure is closely related to Beurling's Criterion, a nice and straightforward sufficient condition, which guarantees that an admissible function for a family of curves in $\mathbb{R}^{2}$ is extremal for its module. Badger [8] generalized Beurling's criterion to $\mathbb{R}^{n}$ making it necessary and sufficient for the Fuglede $p$-module of measure systems.

It is well known that in $\mathbb{R}^{n}, n \geq 2$, the module of the family of all rectifiable curves $\Gamma$, in a spherical ring domain $\Omega=R_{a b}$, connecting the two boundary concentric spheres of radii $a, b, 0<a<b<\infty$, is equal to the module of the family of radial curves $\Gamma_{0}$, connecting these boundary spheres, and that $\Gamma_{0}$ is extremal. In the same spirit, the family of concentric spheres of radii $r$, $a<r<b$, separating the boundary spheres is extremal for the module of all Lipschitz separating surfaces in $R_{a b},[29$, 74, 85, 88]. The extremal functions are also known. Finding the extremal function and the $p$-module is, in general, quite a difficult task, possible to be completed only in a few cases. One of the main aims of this paper is extending a relatively less known result by Rodin [69], which provides a method for finding the extremal function that leads to an explicit calculation of the module of a complete extremal family of curves in the plane, to $\mathbb{R}^{n}, n \geq 2$, and to polarizable Carnot groups.

In Section 2 we study applications of 2-modules of families of curves in $\mathbb{R}^{2}$, introduce Rodin's theorem, Beurling's criterion, the notion of extremal curve family, and briefly discuss its existence and uniqueness for curves. We apply Rodin's method to obtain exact formulas for the extremal function and the module of parallel slanted intervals in a parallelogram, and of logarithmic spirals in a ring domain, as well as of their images. We give estimates for the module of a quadrilateral and of a ring domain in terms of directional dilatations. 
In Section 3, we provide extensions of Rodin's theorem [69] to higher dimensions for rather general type of condensers. One of the main results is Theorem 3 which explicitly calculates the $p$-module $M_{p}\left(\Gamma_{0}^{\prime}\right)$ of an extremal family of curves $\Gamma_{0}^{\prime}=f\left(\Gamma_{0}\right)$, where $f$ is a diffeomorphism in $\Omega$ and $\Gamma_{0}=\Gamma_{0}\left(\Omega ; D_{0}, D_{1}\right)$. An analogous result for the module of a system of measures associated with sets separating $D_{0}$ and $D_{1}$ in $\Omega$ is obtained in Theorem 4 . The tools to handle this more difficult case are developed in Section 3.4.2, where we consider special cases of the coarea and change-of-variable formulas. Two typical examples of $\left(\Omega ; D_{0}, D_{1}\right)$ are a cylinder and a spherical ring domain in $\mathbb{R}^{n}$. Using monotonicity property of modules with respect to families of curves we come to some useful inequalities. Turning from the smooth case to more general settings, we discuss several minimal regularity properties of the homeomorphism $f$. In particular, we can assume $f$ to be Sobolev regular and of finite distortion.

After a thorough analysis has been developed on Carnot groups, see e.g., [36, 37, 81, analogous problems can be formulated for these groups. The only locally rectifiable curves on Carnot groups are so-called horizontal curves. To define a ring domain on Carnot groups one can use an analogue of the Euclidean norm which is a homogeneous functions with respect to the anisotropic dilation $\delta_{s}, s>0$, respecting grading of the corresponding Lie algebras. Unfortunately, the radial curves $\delta_{(\cdot)}$ (considered as functions of $s$ ) are not horizontal in general. On the Heisenberg group, Korányi and Reimann [47] found another family of 'radial' curves which are horizontal and orthogonal (in a correct sense) to the spheres defined as the level sets of the homogeneous norm. It was also shown that this family of curves is extremal for the ring domain. The existence of a homogeneous norm and corresponding horizontal family of radial curves was proved for some other classes of Carnot groups in [7], which received the name polarizable Carnot groups. One of our aims in Section 4 is to show that the family of concentric spheres separating two boundaries of the spherical ring domain is also extremal for the module of all separating sets in this ring on polarizable Carnot groups. We extend Theorem 3 to this geometric setting.

\section{RODIN'S THEOREM AND EXTREMALITY IN $\mathbb{R}^{2}$}

The modules of a quadrilateral and of a ring domain, were originally introduced and studied in the works of Grötzsch, Teichmüller, Ahfors, and many others [1, 31, 41, 50, 77] in the first half of the $20^{\text {th }}$ century. It is natural to assume that these concepts have provided some of the inspiration behind the development of the notion of the extremal length of a family of curves by Beurling and published in a joint work with Ahlfors [3] in 1950. The module of a family of curves is defined as the reciprocal of the extremal length of that 
family. These concepts and their generalizations have become a powerful tool in the study of a wide range of function-theoretic properties of domains in the complex plane, space, and on general metric measure spaces.

2.1. Extremal functions and extremal families of curves. First, we reformulate Definition 1 for the special case of 2-module of a family of curves in $\mathbb{R}^{2}$, state Beurling's criterion, discuss extremal functions, and introduce the notion of extremal families of curves.

Definition 2. Let $\Omega$ be a domain in $\mathbb{R}^{2}$, and let $\rho: \mathbb{R}^{2} \rightarrow \mathbb{R}$ be a non-negative, measurable function. Let $\Gamma$ be a family of locally rectifiable curves in $\Omega$. We say that $\rho$ is an admissible function for $\Gamma$ if

$$
\int_{\gamma} \rho d s \geq 1, \quad \text { for any } \gamma \in \Gamma,
$$

where $\gamma$ is parametrized by arc-length s. Then the quantity

$$
M_{2}(\Gamma):=\inf \left\{\int_{\Omega} \rho^{2}(x) d m: \text { among admissible } \rho\right\},
$$

where $m$ is the Lebesgue measure in $\mathbb{R}^{2}$, is called the 2-module of $\Gamma$. If the equality in (3) is attained for some function $\rho_{0}$, then this function is called extremal for the module problem $M_{2}(\Gamma)$.

The extremal function is essentially unique when it exists (see, e.g., [26]), and it exists when we possibly exclude some subfamilies of curves of vanishing module, see [25] or Proposition [6, item (7).

In what follows, we also use conformal invariance, monotonicity and subadditivity of the 2-module, properties derived in e.g. [1, 26, 63, 78,

Definition 3. Let $\rho_{0}$ be an extremal function for $M_{2}(\Gamma)$. We say that $\Gamma_{0} \subset$ $\Gamma$ is an extremal family of curves for $M_{2}(\Gamma)$ if it satisfies the following two conditions:

$$
\int_{\gamma} \rho d s=1, \quad \text { for } M_{2} \text { - almost all } \gamma \in \Gamma_{0} \text {, and } M_{2}(\Gamma)=M_{2}\left(\Gamma_{0}\right) .
$$

Below we discuss the existence and uniqueness of extremal families, up to a family of curves of vanishing 2-module .

Let $Q_{a b}=\{(x, t): 0 \leq x \leq a, 0 \leq t \leq b\}$ and let $D_{0}=\{(x, t): 0 \leq$ $x \leq a, t=0\}, D_{1}=\{(x, t): 0 \leq x \leq a, t=b\}$. Let $\Gamma=\Gamma\left(Q_{a b}, D_{0}, D_{1}\right)$ be the family of all curves connecting $D_{0}$ and $D_{1}$. One can show that the function $\rho_{0}=\frac{1}{b}$ is extremal for $M_{2}(\Gamma)$, and therefore $M_{2}(\Gamma)=\frac{a}{b}$, see e.g., [1, 50]. Also, the family $\Gamma_{0} \subset \Gamma$ of vertical segments connecting $D_{0}$ and $D_{1}$ satisfies the properties (4) and therefore is extremal for $M_{2}(\Gamma)$. The extremal 
family for the module $M_{2}(\Gamma)$ in $Q_{a b}$, is unique up to a family of curves of vanishing 2 -module. The family $\Gamma \backslash \Gamma_{0}$, has $\rho_{0}$ as an extremal function, too. However, there is no extremal family for $M_{2}\left(\Gamma \backslash \Gamma_{0}\right)$. Consider any conformal mapping $f: Q_{a b} \rightarrow Q$. The image $f\left(\Gamma_{0}\right)$ is an extremal family for the 2-module $M_{2}\left(\Gamma\left(Q ; f\left(D_{0}\right), f\left(D_{1}\right)\right)\right)$ of the family of curves connecting $f\left(D_{0}\right)$ and $f\left(D_{1}\right)$ in $Q$, and the extremal function is $\rho=\frac{1}{b}\left|f^{\prime}\right|$.

Consider the square $Q_{11}$, and the family of all curves $\Gamma^{\star}$ connecting the horizontal sides or the vertical sides of $Q_{11}$. Let $\Gamma_{0}$ be the family of vertical segments connecting the horizontal sides, and $\Sigma_{0}$ be the family of horizontal segments connecting the vertical sides of $Q_{11}$, (see [8]). The extremal function for $M_{2}\left(\Gamma^{\star}\right)$ is $\rho_{0}=1$, and each of $\Gamma_{0}, \Sigma_{0}$, and $\Gamma_{0} \cup \Sigma_{0}$ is an extremal family for $M_{2}\left(\Gamma^{\star}\right)$. In this example the extremal family for $M_{2}\left(\Gamma^{\star}\right)$ is not uniquely determined (up to a set of curves of vanishing 2-module). In this paper we consider extremal family of curves that are unique up to a set of curves of 2-module equal to 0 .

Below we state Beurling's extremaility criterion, formulated by Ahlfors, [1, Theorem 4-4] and cited as Beurling's unpublished work. It provides a sufficient condition guaranteeing that an admissible function for a family of curves in the plane is extremal for its module.

Theorem A (Beurling's Extremality Criterion). Let $\Gamma$ be a family of curves in a domain $\Omega \subseteq \mathbb{R}^{2}$ and let $\rho_{0}$ be an admissible function for $\Gamma$. Suppose that there exists a subfamily $\Gamma_{0}$ in $\Omega$, such that

- $\Gamma_{0} \subseteq \Gamma$;

- $\int_{\gamma} \rho_{0} d s=1$ for every $\gamma \in \Gamma_{0}$;

- For all real valued functions $g \in L^{2}(\Omega)$, the condition $\int_{\gamma} g d s \geq 0$ for all $\gamma \in \Gamma_{0}$, implies

$$
\int_{\Omega} \rho_{0} g d m \geq 0
$$

Then $\rho_{0}$ is extremal for $M_{2}(\Gamma)$ and

$$
M_{2}\left(\Gamma_{0}\right)=M_{2}(\Gamma)=\int_{\Omega} \rho_{0}^{2} d m
$$

Remark 1. As one can see from the proof of Theorem $A$ [1], one needs to consider only the case $g=\rho-\rho_{0}$ for all admissible $\rho$ for $M_{2}(\Gamma)$ to claim extremality of $\rho_{0}$. Indeed, (5) implies $\int_{\Omega} \rho_{0}^{2} d m \leq \int_{\Omega} \rho \rho_{0} d m$. Squaring both sides, applying the Cauchy-Schwarz inequality $\left(\int_{\Omega} \rho_{0}^{2} d m\right)^{2} \leq \int_{\Omega} \rho^{2} d m \int_{\Omega} \rho_{0}^{2} d m$, implies $\int_{\Omega} \rho_{0}^{2} d m \leq \int_{\Omega} \rho^{2} d m$, for all admissible $\rho$. Therefore $M_{2}(\Gamma)=\int_{\Omega} \rho_{0}^{2} d m$ and $\rho_{0}$ is extremal. 
In view of this criterion, $\Gamma_{0}$ is an extremal family of curves for $M_{2}(\Gamma)$. Let us observe that the practical verification of admissibility of $\rho_{0}$ for the entire family $\Gamma$ is perhaps the most difficult part of this criterion although the other parts are equally important. As a supporting argument, consider the following example.

Example 1. Let $\Omega=Q_{1, h \sin \theta}=[0,1] \times[0, h \sin \theta] \subset \mathbb{C}, \theta \in(0, \pi / 2]$, and let $\Omega^{\prime}=f(\Omega)$, where

$$
f(x, t)=(x+t \cot \theta, t), \quad t \in[0, h \sin \theta], \quad x \in[0,1], \quad h>0, \quad \theta \in(0, \pi / 2) .
$$

Then $\Omega^{\prime}$ is the parallelogram in $\mathbb{C}$ with the vertices at $0,1,1+h e^{i \theta}, h e^{i \theta}$. Define the family of locally rectifiable curves $\Sigma$ in $\Omega$ joining the opposite vertical sides of $\Omega$ and by $\Sigma_{0}$ the subfamily of horizontal intervals. Let $\Sigma^{\prime}=f(\Sigma)$ and $\Sigma_{0}^{\prime}=f\left(\Sigma_{0}\right)$. One has $M_{2}\left(\Sigma_{0}^{\prime}\right)=M_{2}\left(\Sigma_{0}\right)=M_{2}(\Sigma)=h \sin \theta$, see [2, Lemma, Page 35]. At the same time, see [4],

$$
M_{2}\left(\Sigma^{\prime}\right)=\frac{\mathbf{K}^{\prime}}{\mathbf{K}}\left(r_{\theta / \pi}\right)
$$

where

$$
r_{\theta / \pi}=\mu^{-1}\left(\frac{\pi h}{2 \sin \theta}\right), \quad \mu(r)=\frac{\pi}{2 \sin \theta} \frac{\mathbf{F}\left(\frac{\theta}{\pi}, 1-\frac{\theta}{\pi} ; 1 ; 1-r^{2}\right)}{\mathbf{F}\left(\frac{\theta}{\pi}, 1-\frac{\theta}{\pi} ; 1 ; r^{2}\right)} .
$$

Here $\mathbf{K}$ and $\mathbf{K}^{\prime}$ are complete elliptic integrals, and $\mathbf{F}$ means the Gaussian hypergeometric function ${ }_{2} F_{1}$. In this example, we see that $M_{2}\left(\Sigma_{0}^{\prime}\right)$ is rather simple expression, whereas the calculation of the module of the larger family of curves $\Sigma^{\prime}$ is a much harder task and requires explicit conformal maps based on the Weierstrass $\wp$-function.

Due to the monotonicity property we can estimate the module of a family of curves using a convenient subset. For example, the inequality $M_{2}\left(\Sigma_{0}^{\prime}\right) \leq$ $M_{2}\left(\Sigma^{\prime}\right)$ gives an interesting lower estimate for the elliptic integrals

$$
h \sin \theta \leq \frac{\mathbf{K}^{\prime}}{\mathbf{K}}\left(r_{\theta / \pi}\right) .
$$

The modules for $\Sigma_{0}^{\prime}$ and $\Sigma^{\prime}$ coincide only if $\theta=\pi / 2$, and in this case $\Sigma_{0}$ is the extremal family for $\Sigma$. In view of Theorem $A$, this means that the function $\rho_{0}(x) \equiv 1$ is extremal for $\Sigma_{0}$, but is not admissible for $\Sigma$ whereas other conditions of Beurling's criterion remain true.

2.2. Rodin's theorem and applications. First, we state Rodin's theorem which provides an explicit method for calculating the extremal functions and the module of a family of curves. Later, we obtain upper and lower estimates for the conformal module of quadrilaterals and doubly connected domains, in terms of directional dilatations, see Definition 4 . 
Let $f$ be a smooth, orientation preserving homeomorphism of $Q_{1 b}$ onto a region $Q \in \mathbb{R}^{2}$, such that the Jacobi matrix exists and its determinant $J_{f}$ is strictly positive. Let $\Gamma_{0}$ be the family of vertical intervals $v_{x}(t)=\{(x, t): t \in$ $[0, b] ; x \in[0,1]$ is fixed $\}$, and let $c_{x}(t)=f\left(v_{x}(t)\right) \in Q$. Thus, the image of $\Gamma_{0}$ is $f\left(\Gamma_{0}\right)=\left\{c_{x}:[0, b] \rightarrow Q, x \in[0,1]\right\}$.

Theorem B (Rodin's Theorem, [69]). Let

$$
\ell(x)=\int_{0}^{b} \frac{\left|\dot{c}_{x}\right|^{2}}{J_{f}} d t, \quad x \in[0,1],
$$

where $\dot{c}_{x}=\frac{\partial}{\partial t} c_{x}(t)$. Then

$$
\rho_{0}(y)=\frac{1}{\ell(x)}\left(\frac{\left|\dot{c}_{x}\right|}{J_{f}}\right) \circ f^{-1}(y), \quad(x, t) \in Q_{1 b}, \quad y=f(x, t) \in Q,
$$

is the extremal function for the 2-module of the family $f\left(\Gamma_{0}\right)$ and

$$
M_{2}\left(f\left(\Gamma_{0}\right)\right)=\int_{Q} \rho_{0}^{2}(y) d y=\int_{0}^{1} \ell^{-1} d x
$$

To prove Theorem $\mathrm{B}$, one shows that $\rho_{0}$ in (6) satisfies conditions of Theorem A. The computation of $M_{2}\left(f\left(\Gamma_{0}\right)\right)=\int_{\Omega} \rho_{0}^{2} d m$ leads to (7).

The above result was used by Rodin and Warschawski to characterize the boundary behavior of conformal maps, see, e.g., [70, 71].

If a quadrilateral $Q$ is a conformal image of the rectangle $Q_{1 b}$, and if $\Gamma=$ $\Gamma\left(Q_{1 b} ; D_{0}, D_{1}\right)$, then $M_{2}(f(\Gamma))=M_{2}(\Gamma)=1 / b$. If $\Sigma$ is the family of curves separating $D_{0}$ and $D_{1}$ in $Q_{1 b}$, then $M_{2}(f(\Sigma)) M_{2}(f(\Gamma))=M_{2}(\Sigma) M_{2}(\Gamma)=1$, see e.g., [1, 41, 50, 78]. Sometimes, families $\Gamma$ and $\Sigma$ are called conjugate.

Let us return back to Example 1 of the parallelogram $\Omega^{\prime}$. As it was observed, the expression for $M_{2}\left(\Sigma^{\prime}\right)$ is difficult and involves some implicit functions and elliptic integrals. Rodin's theorem allows to give some explicit estimates of $M_{2}\left(\Sigma^{\prime}\right)$. Denote by $\Gamma_{\theta}^{\prime}$ the family of parallel slanted intervals connecting the horizontal sides of $\Omega^{\prime}$. By $\Gamma^{\prime}$, we denote the whole family of curves connecting the horizontal sides of $\Omega^{\prime}$, and if $\theta=\pi / 2$, then $\Gamma_{\theta}^{\prime}=\Gamma_{0}$. As it was remarked, $M_{2}\left(\Sigma_{0}^{\prime}\right)=M_{2}\left(\Sigma_{0}\right)=h \sin \theta$ and $M_{2}\left(\Sigma^{\prime}\right) \geq h \sin \theta$ because of monotonicity of $M_{2}$.

\section{Proposition 1.}

$$
M_{2}\left(\Gamma_{\theta}^{\prime}\right)=\frac{\sin \theta}{h}, \quad M_{2}\left(\Gamma_{\theta}^{\prime}\right) M_{2}\left(\Sigma_{0}^{\prime}\right)=\sin ^{2} \theta,
$$

and

$$
\left|M_{2}\left(\Sigma^{\prime}\right)-M_{2}\left(\Sigma_{0}^{\prime}\right)\right| \leq h \frac{\cos ^{2} \theta}{\sin \theta}
$$


Proof. Applying Theorem B we have

$$
M_{2}\left(\Gamma_{\theta}^{\prime}\right)=\int_{0}^{1} \frac{d x}{\int_{0}^{h \sin \theta} \frac{\left|f_{t}\right|^{2}}{J_{f}} d t}=\frac{\sin \theta}{h}, \quad M_{2}\left(\Sigma_{0}^{\prime}\right)=\int_{0}^{h \sin \theta} \frac{d y}{\int_{0}^{1} \frac{\left|f_{x}\right|^{2}}{J_{f}} d x}=h \sin \theta,
$$

because $\left|f_{t}\right|^{2}=1+\cot ^{2} \theta, J_{f}=1$ and $\left|f_{x}\right|^{2}=1$. This implies (8). By the monotonicity property of the module of a family of curves, we have

$$
M_{2}\left(\Sigma_{0}^{\prime}\right) \leq M_{2}\left(\Sigma^{\prime}\right) \leq\left(M_{2}\left(\Gamma_{\theta}^{\prime}\right)\right)^{-1}
$$

Since $M_{2}\left(\Sigma_{0}^{\prime}\right)=h \sin \theta$, using (8) we obtain (9) .

Remark 2. Of course, the shear map in Example 1 is a quasiconformal homeomorphism with the maximal real dilatation

$$
K(\theta)=1+\frac{1}{2} \cot ^{2} \theta+\frac{1}{2} \cot \theta \sqrt{4+\cot ^{2} \theta} \geq 1 .
$$

So the estimate $M_{2}\left(\Sigma^{\prime}\right) \leq K h \sin \theta$ holds trivially. However, $K(\theta) \geq 1 / \sin ^{2} \theta$ and the estimate in Proposition 9 is better for this map.

Applying Rodin's method, we obtain useful estimates for the conformal modules of quadrilaterals and ring domains by using the monotonicity property of 2-modules of curves.

The vertices of $Q_{a b}$ are usually assigned a specific order, namely $(0,0),(a, 0)$, $(a, b)$ and $(b, 0)$, and the segments $D_{0}$ and $D_{1}$ are called its $a$-sides, thus these are the sides connecting the first and the second and the third and the fourth vertices. By a quadrilateral $Q$ we understand a conformal image $f\left(Q_{a b}\right)$ of a rectangle $Q_{a b}$ with a fixed order of vertices, the images of the vertices of $Q_{a b}$ and thus with identified $a$-sides, the images of $D_{0}$ and $D_{1}$. The conformal module of the quadrilateral $Q$ with the above assigned order of vertices and $a$-sides is defined to be

$$
M(Q)=b / a .
$$

Thus, $M(Q)=M_{2}(\Sigma)=M\left(Q_{a b}\right)=b / a$, where $\Sigma=\Sigma\left(Q_{a b} ; D_{0}, D_{1}\right)$ is the family of curves separating $D_{0}$ and $D_{1}$ in $Q_{a b}$. It is well-known that $M(Q)$ can be obtained by minimizing the Dirichlet integral min $\iint_{Q}|\nabla v|^{2} d x d y$, for a set of admissible for the capacity functions, see Definition [14, and is equal to the capacity of the condenser determined by the domain $Q$ with the plates which are the images of the vertical sides of $Q_{a b}$.

The parallelogram $P(\varepsilon)$ with vertices $0,1,1+\varepsilon+i b, \varepsilon+i b$ is the image of $Q_{1 b}$ under the sheer transformation $f(x, t)=(x+\varepsilon t, t)$. The properties of its conformal module, $M\left(P_{\varepsilon}\right)$, have been extensively studied in many papers. For example, Reich [65] showed that $M\left(P_{\varepsilon}\right)$ is a convex non-decreasing function of $\varepsilon$, using Steiner symmetrization. This result was extended in a paper of Dubinin 
and Vuorinen [20] who studied the change of conformal modules of polygonal quadrilaterals under some transformations. Properties of conformal modules of polygonal quadrilaterals, including parallelograms, have been investigated using hypergeometric functions and numerical methods in [4, 35, 42, 64] and many others, see the references therein.

Applying Rodin's result, we obtain the following inequality that provides an estimate for the rate of convergence of $M\left(P_{\varepsilon}\right)$ to $M\left(Q_{1 b}\right)$, as $\varepsilon \rightarrow 0$.

\section{Proposition 2.}

$$
\left|M(P(\varepsilon))-M\left(Q_{1 b}\right)\right| \leq \frac{\varepsilon^{2}}{b} .
$$

The proof is done in analogy to Proposition 1 ,

Now we turn to ring domains. A ring domain $R$ is a conformal image of the annulus $R_{a b}=\{z: a \leq|z| \leq b\}$. The conformal module $M(R)$ of $R$ is defined as

$$
M(R)=\frac{\log b / a}{2 \pi} .
$$

Extremal properties of modules of doubly connected regions, or ring domains, have been originally studied in the works of Teichmüller and Grötzsch, see [50] for some results and history of the topic. The concept has played an essential role in the development of the theory of plane quasiconformal mappings and is naturally connected to the 2-module of families of curves.

If $\Gamma$ is a family of curves connecting the boundary circles $S_{a}$ and $S_{b}$, let $\Sigma$ be the family of curves separating $S_{a}$ and $S_{b}$ in $R_{a b}$. Denote by $f(\Gamma)$ and $f(\Sigma)$ their images under a conformal map $f$ that maps $R_{a b}$ onto $R$. One can show that

$$
M_{2}(f(\Gamma))=M_{2}(\Gamma)=\frac{2 \pi}{\log b / a}
$$

and that

$$
M_{2}(f(\Sigma)) M_{2}(f(\Gamma))=M_{2}(\Sigma) M_{2}(\Gamma)=1 .
$$

Therefore, $M(R)=M_{2}(\Sigma)=M_{2}(f(\Sigma))$. Thus, the conformal module can be defined as the 2-module of the family of curves $\Sigma=\Sigma\left(R_{a b} ; S_{a}, S_{b}\right)$ separating the boundaries $S_{a}$ and $S_{b}$ in the annulus $R_{a b}$.

We denote by $\Gamma_{0}$ the family of radial intervals connecting $S_{a}$ and $S_{b}$ and by $\Sigma_{0}$ the family of concentric circles separating $S_{a}$ and $S_{b}$. The families of curves $\Sigma_{0}, f\left(\Sigma_{0}\right), \Gamma_{0}, f\left(\Gamma_{0},\right)$ are extremal families for the corresponding module problems.

Now, let $f: R_{1 b} \rightarrow R^{\prime}$, be an orientation preserving homeomorphism (not necessarily conformal) defined in a neighborhood of $R_{1 b}$. A point in $R_{1 b}$ is called regular following [15], if $f$ is differentiable at this point and the Jacobian 
$J_{f}$ is strictly positive. At a regular point $z=r e^{i \theta} \in R_{1 b}$, the complex dilatation of $f$ is $\mu_{f}=\frac{f_{\bar{z}}}{f_{z}}$, and the Jacobian is $J_{f}=\left|f_{z}\right|^{2}-\left|f_{\bar{z}}\right|^{2}=\left|f_{z}\right|^{2}\left(1-\left|\mu_{f}\right|^{2}\right)$.

Below we introduce the directional dilatation of $f$ in the direction $\alpha$, at a regular point. This concept was studied by Andreian-Cazacu [15] for the purpose of generalizing the class of $K$-quasiconformal mappings.

Definition 4. The directional dilatation of $f$ in the direction $\alpha \in \mathbb{R}$, is defined as

$$
D_{f, \alpha}=\frac{\left|1+e^{-2 i \alpha} \mu_{f}\right|^{2}}{1-\left|\mu_{f}\right|^{2}} \text {. }
$$

Denote by $f_{r}=\frac{\partial}{\partial r} f\left(r e^{i \theta}\right)$ and $f_{\theta}=\frac{\partial}{\partial \theta} f\left(r e^{i \theta}\right)$. Then $f_{r}=e^{i \theta}\left(f_{z}+e^{-2 i \theta} f_{\bar{z}}\right)$ and $f_{\theta}=i r e^{i \theta}\left(f_{z}-e^{-2 i \theta} f_{\bar{z}}\right)$. Hence, $D_{f, \theta}=\frac{\left|f_{r}\right|^{2}}{J_{f}}, \quad D_{f, \theta+\frac{\pi}{2}}=\frac{\left|f_{\theta}\right|^{2}}{r^{2} J_{f}}$. We assume that $0 \leq \theta<2 \pi$.

Let $\Gamma_{0}^{\prime}, \Gamma^{\prime}, \Sigma_{0}^{\prime}$, and $\Sigma^{\prime}$ be the images of $\Gamma_{0}, \Gamma, \Sigma_{0}$, and $\Sigma$ under $f$, respectively. Below we state results concerning modules of images under $f$ of radial segments and concentric circles in an annulus and the corresponding extremal functions. For simplicity we assume that $f$ is an orientation preserving $C^{1}$-smooth homeomorphism but the results can be extended, after careful justification, to mappings of exponentially integrable finite distortion and $\mu$-homeomorphisms, which are locally absolutely continuous, see [14].

Proposition 3. The 2-module of the family of curves $\Gamma_{0}^{\prime}$ is calculated as

$$
M_{2}\left(\Gamma_{0}^{\prime}\right)=\int_{0}^{2 \pi}\left(\int_{1}^{b} \frac{D_{f, \theta}}{r} d r\right)^{-1} d \theta
$$

The extremal function for $M_{2}\left(\Gamma_{0}^{\prime}\right)$ is given by

$$
\rho_{0}=\frac{\frac{D_{f, \theta}}{r}}{\left|f_{r}\right| \int_{1}^{b} \frac{D_{f, \theta}}{r} d r} \circ f^{-1} .
$$

Proof. For $0 \leq \theta<2 \pi$, denote by $\gamma_{\theta}$ the radial segment connecting the concentric circles $|z|=1$ and $|z|=b$, making angle $\theta$ with the $x$-axis, and by $\gamma_{\theta}^{\prime}$ its image under $f$. We show that $\rho_{0}$ satisfies the conditions of Theorem A. Indeed, $\int_{\gamma_{\theta}^{\prime}} \rho_{0} d s=1$. Next, let $\rho$ be any admissible function for $M_{2}\left(\Gamma_{0}^{\prime}\right)$. Then $\int_{\gamma_{\theta}^{\prime}}\left(\rho-\rho_{0}\right) d s \geq 0$, and therefore, $\int_{1}^{b}\left(\rho-\rho_{0}\right) \circ f\left|f_{r}\right| d r \geq 0$. Hence,

$$
\int_{R^{\prime}} \rho_{0}\left(\rho-\rho_{0}\right) d m=\int_{0}^{2 \pi}\left(\int_{1}^{b} \frac{D_{f, \theta}}{r} d r\right)^{-1} \int_{1}^{b}\left(\rho-\rho_{0}\right) \circ f\left|f_{r}\right| d r d \theta \geq 0 .
$$


By Theorem A, $M_{2}\left(\Gamma_{0}^{\prime}\right)=\int_{R^{\prime}} \rho_{0}^{2} d m$. A simple calculation leads to (10).

The theorem below was proved in [14].

Proposition 4. The 2-module of the family of curves $\Sigma_{0}^{\prime}$ is calculated as

$$
M_{2}\left(\Sigma_{0}^{\prime}\right)=\int_{1}^{b}\left(\int_{0}^{2 \pi} D_{f, \theta+\frac{\pi}{2}} d \theta\right)^{-1} \frac{d r}{r},
$$

and the extremal function is

$$
\rho_{0}=\frac{D_{f, \theta+\frac{\pi}{2}}}{\left|f_{\theta}\right| \int_{0}^{2 \pi} D_{f, \theta+\frac{\pi}{2}}} \circ f^{-1} .
$$

From the monotonicity property, Propositions 3 and 4 , we have $M_{2}\left(\Sigma_{0}^{\prime}\right) \leq$ $M_{2}\left(\Sigma^{\prime}\right) \leq\left(M_{2}\left(\Gamma_{0}^{\prime}\right)\right)^{-1}$. Denote by $M\left(R^{\prime}\right)$ the conformal module of $R^{\prime}$. Since $M\left(R^{\prime}\right)=M_{2}\left(\Sigma^{\prime}\right)$, this leads to the following useful estimate for $M\left(R^{\prime}\right)$, in terms of directional dilatations.

Theorem 1. For the conformal module $M\left(R^{\prime}\right)$ of $R^{\prime}$ we have

$$
\int_{1}^{b}\left(\int_{0}^{2 \pi} D_{f, \theta+\frac{\pi}{2}} d \theta\right)^{-1} \frac{d r}{r} \leq M\left(R^{\prime}\right) \leq\left(\int_{0}^{2 \pi}\left(\int_{1}^{b} \frac{D_{f, \theta}}{r} d r\right)^{-1} d \theta\right)^{-1} .
$$

Observe that the upper and lower estimates are conformally invariant. Applying the Cauchy-Schwarz inequality leads to

$$
\int_{1}^{b}\left(\int_{0}^{2 \pi} D_{f, \theta+\frac{\pi}{2}} d \theta\right)^{-1} \frac{d r}{r} \leq M\left(R^{\prime}\right) \leq \frac{1}{(2 \pi)^{2}} \int_{R_{1 b}} \frac{D_{f, \theta}}{|z|^{2}} d m .
$$

Such estimates have been used in e.g., [11, 12, 13, 14, 32, 49] and others. To obtain (11) directly, one usually applies the length-area method, which involves integrating over curves and using Fubini's theorem, see Volkovyskii [87], Reich and Walczak [66], Gutlyanskii and Martio [32] and others (the history and equivalence of such estimates under the assumption that $f$ is quasiconformal, have been discussed in [10]).

Now we derive expressions for the modules of a family of logarithmic spirals and their images under a smooth homeomorphism $f$ in terms of directional dilatations. Let $\beta \in \mathbb{R}$. Consider $f: R_{1 b} \rightarrow R_{1 b}, f\left(r e^{i \theta}\right)=|z| e^{i(-\beta \log |z|+\theta)}$, which maps the radial segments in $R_{1 b}$ into portions of logarithmic spirals in $R_{1 b}$ of inclination $\beta$. Denote by $\Gamma_{\beta}=\bigcup_{\theta \in[0,2 \pi)}\left\{z: z=r e^{i(-\beta \log r+\theta)}, 1<r<b\right\}$ and by $\Gamma_{\beta}^{\prime}$ its $f$-image. Applying previous results and using Rodin's theorem, we obtain the following useful for the applications result.

Proposition 5. The 2-module of the family of logarithmic spirals $\Gamma_{\beta}$ is

$$
M_{2}\left(\Gamma_{\beta}\right)=\frac{2 \pi}{\left(1+\beta^{2}\right) \log b .}
$$


Also

$$
M_{2}\left(\Gamma_{\beta}^{\prime}\right)=\int_{0}^{2 \pi}\left(\int_{1}^{b}\left(1+\beta^{2}\right) D_{f, \theta_{0}} \frac{d r}{r}\right)^{-1} d \theta
$$

where $\theta_{0}=-\beta \log r+\theta+\arctan \beta$.

Proof. Let $z=r e^{i \theta}$ represent the polar coordinates of $z$ in the annulus $R_{1 b}$. Then $f_{r}=e^{i(-\beta \log r+\theta)}(1-i \beta)$. Thus $\left|g_{r}\right|^{2}=\left(1+\beta^{2}\right)$. In addition $J_{f}=1$. From Proposition 3 , it follows that the extremal function for $M_{2}\left(\Gamma_{\beta}\right)$ is

$$
\rho_{0} \circ f\left(r e^{i \theta}\right)=\frac{\sqrt{1+\beta^{2}}}{r \int_{1}^{b}\left(1+\beta^{2}\right) \frac{d r}{r}}=\frac{1}{\sqrt{1+\beta^{2}}(r \log b)} .
$$

Thus

$$
M_{2}\left(\Gamma_{\beta}\right)=\int_{R_{1 b}} \rho_{0}^{2} d m=\int_{1}^{b} \int_{0}^{2 \pi} \frac{1}{1+\beta^{2}} \frac{1}{(r \log b)^{2}} r d r d \theta=\frac{2 \pi}{\left(1+\beta^{2}\right) \log b},
$$

which proves (12).

Consider the mapping $g=f\left(r e^{i(-\beta \log r+\theta)}\right)$ of the annulus $R_{1 b}$ in the polar coordinates $Q=\{(r, \theta): 1 \leq r \leq b, 0 \leq \theta<2 \pi\}$ on $Q^{\prime}$. Then $M_{2}\left(\Gamma_{\beta}^{\prime}\right)=$ $\int_{0}^{2 \pi}\left(\int_{1}^{b} \frac{\left|g_{r}\right|^{2}}{J_{g}} d r\right)^{-1} d \theta$. Since $\left|g_{r}\right|^{2}=\left|f_{z}+f_{\bar{z}} e^{i \theta_{0}}\right|^{2}, J_{g}=r J_{f}$, and by Definition 4, we obtain (12).

Since $M_{2}\left(\Sigma^{\prime}\right) \leq\left(M_{2}\left(\Gamma_{\beta}^{\prime}\right)\right)^{-1}$, after applying the Cauchy Schwarz inequality one obtains estimates for $M_{2}\left(\Sigma^{\prime}\right)$, which depend on $\beta$ :

$$
M_{2}\left(\Sigma^{\prime}\right) \leq \frac{1+\beta^{2}}{(2 \pi)^{2}} \int_{R_{1 b}} D_{f, \theta_{0}} \frac{d m}{|z|^{2}} .
$$

Since $M_{2}\left(\Sigma^{\prime}\right)=M\left(R^{\prime}\right)$ the above inequality provides a conformally invariant upper estimate for $M\left(R^{\prime}\right)$, which is more general than the one obtained in (11). After simple calculations, it can be shown that it is equivalent to the one obtained in [14, Corollary 3.4].

\section{RODIN'S THEOREM IN EUCLIDEAN SPACES}

3.1. Module of a system of measures in Euclidean spaces. The module of a systems of measures, introduced in Definition 1, has the following properties, based on [25, Chapter 1]:

Proposition 6. Let $(X, \mathfrak{M}, m)$ be an abstract measure space where $m$ is a fixed measure defined on a subalgebra of $\mathfrak{M}$. Denote by $\mathcal{M}$ the system of all measures $\mu$ in $X$, whose domains of definition contain $\mathfrak{M}$ and by $\bar{\mu}$ the completion of the measure $\mu$. Then the following properties hold

(1) $M_{p}(E) \leqslant M_{p}\left(E^{\prime}\right)$ if $E \subset E^{\prime}$ and $E, E^{\prime} \subset \mathcal{M}$; 
(2) $M_{p}(E) \leqslant \sum_{i=1}^{\infty} M_{p}\left(E_{i}\right)$ if $E=\bigcup_{i=1}^{\infty} E_{i}$, and $E_{i} \subset \mathcal{M}$;

(3) If $A \subset X$ and $\bar{m}(A)=0$, then $\bar{\mu}(A)=0$ for $M_{p}$-a.a. $\mu \in \mathcal{M}$;

(4) If $\rho \in L^{p}(X, \bar{m})$, then $\rho$ is $\bar{\mu}$-integrable for $M_{p}$-a.a. $\mu \in \mathcal{M}$;

(5) If $\left\|\rho_{i}-\rho\right\|_{L^{p}(X, \bar{m})} \rightarrow 0$, then there is a subsequence $\rho_{i_{j}}$, such that

$$
\int_{X}\left|\rho_{i_{j}}-\rho\right| d \bar{\mu} \rightarrow 0 \quad \text { for } M_{p} \text {-a.e. } \mu \in \mathcal{M}
$$

(6) Let $E \subset \mathcal{M}$. Then $M_{p}(E)=0$, if and only if, there exists a nonnegative function $\rho \in L^{p}(X, m)$, such that

$$
\int_{X} \rho d \mu=+\infty \quad \text { for every } \mu \in E
$$

(7) If $p>1$ and $E \subset \mathcal{M} \backslash\{\mu \equiv 0\}$, then there exists a non-negative function $\rho$, such that

$$
\int_{X} \rho^{p} d m=M_{p}(E), \quad \text { and } \int_{X} \rho d \mu \geqslant 1 \text { for } M_{p} \text {-a.e. } \mu \in E ;
$$

(8) For the measures, which are restrictions of the Hausdorff measure to compact sets, the following is true: if $p \geqslant 2, E_{1} \subset E_{2} \subset \cdots$ are sets of complete measures and $E=\bigcup E_{i}$, then

$$
M_{p}(E)=\lim _{i \rightarrow \infty} M_{p}\left(E_{i}\right) .
$$

Badger, see [8], extended Beurling's criterion (Theorem A) to Fuglede's $p$ module of systems of measures, as a sufficient and necessary condition. Let $X=\mathbb{R}^{n}$, let $\mathfrak{M}$ be a Borel $\sigma$-algebra in the topology defined by the Euclidean metric, and let $m$ be the Lebesgue measure.

Theorem 2 ([8]). Let $E$ be a measure system in $\mathbb{R}^{n}$ and let $\rho$ be an admissible function for $E$ such that $\rho \in L^{p}\left(\mathbb{R}^{n}\right), 1<p<\infty$. Then $\rho_{0}$ is the extremal function for the $p$-module of $E$ if and only if, there exists a measure system $F$ such that

- $M_{p}(F \cup E)=M_{p}(E)$;

- $\int_{\mathbb{R}^{n}} \rho_{0} d \nu=1$ for every $\nu \in F$;

- For all real valued functions $g \in L^{p}\left(\mathbb{R}^{n}\right)$ the condition $\int_{\mathbb{R}^{n}} g d \nu \geq 0$, for all $\nu \in F$, implies $\int_{\mathbb{R}^{n}} g \rho_{0}^{p-1} d m \geq 0$.

Analogous theorem holds for $p=1$.

Remark 3. In the same manner, as in the plane, one can see from the proof of Theorem 2, that we only need to consider $g=\rho-\rho_{0}$ to get extremality for $\rho_{0}$ and the systems of measures $E$. 
3.2. Rodin's theorem for families of connecting curves in a condenser. Here we state an extension of Rodin's result [69, Theorem 14] to the $p$-module of families of curves in $\mathbb{R}^{n}$, connecting the two plates $D_{0}$ and $D_{1}$ of a condenser $\left(\Omega ; D_{0}, D_{1}\right)$, introduced at the beginning of Section 1 .

Recall that for any Borel set $B$, we use the Hausdorff measure defined by $H^{d}(B)=\lim _{\delta \rightarrow 0} H_{\delta}^{d}(B)$, where

$$
H_{\delta}^{d}(B)=\inf \left\{\sum_{i=1}^{\infty}\left(\operatorname{diam} U_{i}\right)^{d}: U_{i} \text { are open, } \bigcup_{i=1}^{\infty} U_{i} \supseteq B, \operatorname{diam} U_{i}<\delta\right\} .
$$

We observe that the $n$-dimensional Lebesgue measure coincides with the $H^{n}$ Hausdorff measure in $\mathbb{R}^{n}$ for all $n$, up to a constant which we choose to be 1 . The number $d$ is called the Hausdorff dimension of $B$.

Let $D \subset \mathbb{R}^{n}$ be an $(n-1)$-Hausdorff dimensional compact and let $(x, t) \mapsto$ $\mathbf{u}(x, t)$, where $x \in D$ and $t \in[a, b]$, be an embedding that parametrizes a condenser $\left(\Omega ; D_{0}, D_{1}\right)$ in $\mathbb{R}^{n}$, so that $D_{0}=\mathbf{u}(x, a)$ and $D_{1}=\mathbf{u}(x, b)$. We assume that $\mathbf{u}=\left(u_{1}, \ldots, u_{n}\right)$ is a $C^{1}$-smooth homeomorphism in $D \times[a, b]$ with a positive Jacobian $J_{\mathbf{u}}$. Let $f$ be a $C^{1}$-smooth homeomorphism of $\Omega$ onto $\Omega^{\prime} \subset \mathbb{R}^{n}$, such that the Jacobian $J_{f}$ is strictly positive in $\Omega$. Denote by $I_{f}=J_{\mathbf{u}} J_{f}$.

Let $\Gamma_{0}$ be the family of curves

$$
v_{x}(t)=\{\mathbf{u}(x, t): t \in[a, b], x \in D \text { is fixed }\},
$$

and let $c_{x}(t)=f\left(v_{x}(t)\right) \subset \Omega^{\prime}$. Then

$$
f\left(\Gamma_{0}\right)=\left\{c_{x}:[a, b] \stackrel{\mathrm{u}}{\longrightarrow} \Omega \stackrel{f}{\longrightarrow} \Omega^{\prime}, x \in D\right\} .
$$

We write $\dot{c}_{x}=\frac{\partial}{\partial t} c_{x}$.

Our generalization of Rodin's theorem is as follows.

Theorem 3. Set $1 / p+1 / q=1, p, q>1$, and let

$$
\ell(x)=\int_{a}^{b}\left(\frac{\left|\dot{c}_{x}\right|}{I_{f}}\right)^{q} I_{f} d t, \quad x \in D .
$$

Then

$$
\rho_{0}(y)=\frac{1}{\ell(x)}\left(\frac{\left|\dot{c}_{x}\right|}{I_{f}}\right)^{\frac{1}{p-1}} \circ f^{-1}, \quad y=f \circ \mathbf{u}(x, t) \in \Omega^{\prime},
$$

is the extremal function for the p-module $M_{p}\left(f\left(\Gamma_{0}\right)\right)$ of the family $f\left(\Gamma_{0}\right)$, where

$$
M_{p}\left(f\left(\Gamma_{0}\right)\right)=\int_{\Omega^{\prime}} \rho_{0}^{p} d y=\int_{D} \ell^{1-p} d H^{n-1}(x) .
$$

$f\left(\Gamma_{0}\right)$ is the complete extremal family of curves for $M_{p}\left(f\left(\Gamma_{0}\right)\right)$. 
The proof of the above theorem is given in Section 3.4.1.

The only result known to us, that computes explicitly the weighted $p$-module of a family of curves in $R^{n}$ and provides the almost extremal function for it, belongs to Ohtsuka, see Theorem 3.4.3 in [61]. The author studies a family of curves in a tube, which are trajectories of a solenoidal vector field. It is clear that, while considering different settings, Ohtsuka's result and Theorem 3, should lead to equivalent formulas, when the settings overlap.

Observe that Theorem 3 applies to the following special cases.

- $\Omega=\{t \in[a, b], x \in D\}$ is a cylinder in $\mathbb{R}^{n}$, where $D$ is a compact set in $\mathbb{R}^{n-1}$ and $\mathbf{u} \equiv \mathrm{id}$. Then, $I_{f}=J_{f}$.

- A spherical ring domain $\Omega=R_{a b}$ in $\mathbb{R}^{n}$ bounded by the concentric spheres $S_{a}$ and $S_{b}$ of radii $a$ and $b$ respectively. Then $D=S_{1}$ is the unit sphere in $\mathbb{R}^{n}, \mathbf{u}(x, t)=t x$, and $I_{f}=t^{n-1} J_{f}$.

- A conical cylinder $\Omega=\left\{(\beta t x, t): x \in D \subset \mathbb{R}^{n-1}, t \in[a, b], \beta>0\right\}$. Then $I_{f}=(\beta t)^{n-1} J_{f}$

3.3. Modules of families of separating sets in a condenser. Let us define separating sets in a topological sense and describe the associated system of measures. Let $D_{0}$ and $D_{1}$ be disjoint compact sets in the closure $\bar{\Omega}$ of a bounded open set $\Omega \subset \mathbb{R}^{n}$. We denote $\Omega^{*}=\Omega \cup D_{0} \cup D_{1}$.

Definition 5. We say that a set $\sigma$ separates $D_{0}$ from $D_{1}$ in $\Omega$ if

- $\sigma \cap \Omega$ is closed in $\Omega$;

- There are disjoint sets $U_{1}$ and $U_{2}$ which are open in $\Omega^{*} \backslash \sigma$ such that $\Omega^{*} \backslash \sigma=U_{1} \cup U_{2}, D_{0} \subset U_{1}$ and $D_{1} \subset U_{2}$.

Let $\Sigma$ denote the class of all sets that separate $D_{0}$ from $D_{1}$. With every $\sigma \subset \Sigma$ we associate a complete measure $\mu$ in the following way: for every Hausdorff $H^{n-1}$-measurable set $A \subset \mathbb{R}^{n}$ we define

$$
\mu(A)=H^{n-1}(A \cap \sigma \cap \Omega) .
$$

From the properties of Hausdorff measure, it is clear that the Borel sets in $\mathbb{R}^{n}$ (here $\sigma \cap \Omega$ is closed in $\Omega$, and therefore, is Borel) are $\mu$-measurable, hence the module $M_{q}(\Sigma)$ of $\Sigma$ is the module of a family of measures $E$ in Definition 1 . We will use the two notations notations $M_{q}(E)$ and $M_{q}(\Sigma)$ interchangeably.

The next theorem states an analogue to Theorem 3 for separating sets. Let $\left(\Omega ; D_{0}, D_{1}\right)$ be a condenser in $\mathbb{R}^{n}$ defined as previously. We define $\Sigma_{0}$ to be a family of sets $\sigma_{t}=\{\mathbf{u}(x, t): x \in D, t \in[a, b]$ is fixed $\}$ that separate the plates $D_{0}=\mathbf{u}(x, a)$ and $D_{1}=\mathbf{u}(x, b)$. Assume that $f$ is $C^{1}$-smooth orientation preserving homeomorphism $\Omega \rightarrow \Omega^{\prime} \subset \mathbb{R}^{n}$. 
Theorem 4. Let $\frac{1}{p}+\frac{1}{q}=1$, and let

$$
\ell(t)=\int_{\sigma_{t}}\left(\mid\left(\nabla\left(t\left(f^{-1}\right)\right) \circ f(\mathbf{u}(x, t)) \mid\right)^{p} I_{f} d \mu .\right.
$$

Then,

$$
\rho_{0}=\frac{1}{\ell\left(t\left(f^{-1}\right)\right.}\left(\mid\left(\nabla\left(t\left(f^{-1}\right)\right) \mid\right)^{1 /(q-1)}\right.
$$

is the extremal metric for the q-module of $\Sigma_{0}^{\prime}=f\left(\Sigma_{0}\right)$, and the q-module is equal to $M_{q}\left(\Sigma_{0}^{\prime}\right)=\int_{\Omega^{\prime}} \rho_{0}^{q} d y=\int_{a}^{b} \ell^{1-q} d t$.

The condition of the regularity for the mapping $f$ and the set $D$ can be relaxed according to the discussion in Section 3.4.2.

Let us give simple but illustrative Examples 2 and 3 of calculation of the extremal functions and families for the module of families of connecting curves and separating sets in the cylinder $\Omega=D \times[a, b]$. Then, Examples 4 and 5 illustrate Theorem 3 for a cylinder and for a spherical ring domain.

Example 2. Let $\Gamma$ be a family of all locally rectifiable curves connecting the base sides $D_{0}$ and $D_{1}$ of the cylinder $\Omega$, and let $\Gamma_{0} \subset \Gamma$ be the family of intervals

$$
v_{x}(t)=\{(x, t): t \in[a, b], x \in D \text { is fixed }\} .
$$

Observe that the function $\rho_{0}(x)=\frac{1}{b-a}, x \in \Omega$ is extremal for $\Gamma$ and

$$
M_{p}(\Gamma)=M_{p}\left(\Gamma_{0}\right)=\frac{H^{n-1}(D)}{(b-a)^{p-1}} .
$$

Example 3. Let $\Sigma$ be a family of Borel sets $\sigma$ separating the base sides $D_{0}=\sigma_{a}$ and $D_{1}=\sigma_{b}$ of the cylinder $\Omega$, and let $\Sigma_{0} \subset \Sigma$ be the family of sets

$$
\sigma_{t}=\{(x, t): x \in D, t \in[a, b] \text { is fixed }\} .
$$

By $E$ we denote the family $H^{n-1}$-Hausdorff measures in $\Omega$ associated with $\sigma \in \Sigma$ and $E_{0} \subset E$ are $H^{n-1}$-Hausdorff measures associated with $\sigma_{t} \in \Sigma_{0}$. It is easy to see that $\varrho_{0}=\frac{1}{H^{n-1}(D)}$ is the extremal function for both $\Sigma$ and $\Sigma_{0} \subset \Sigma$, satisfies all other conditions of Theorem 2 and

$$
M_{q}(\Sigma)=\frac{b-a}{\left(H^{n-1}(D)\right)^{q-1}} .
$$

The classical equality $\left(M_{p}(\Gamma)\right)^{q}\left(M_{q}(\Sigma)\right)^{p}=1$ holds.

Example 4. Now let us calculate the module in the image $\Omega^{\prime}$ of $\Omega$ with $a=0$, $b=r$, under a 'horizontal' shear transform $f$ given by

$$
f(x, t)=\left(x_{1}+\beta t, x_{2}, \ldots, x_{n-1}, t\right) .
$$


Let $\Gamma_{0}$ be as in Theorem 3 and $\Gamma_{0}^{\prime}=f\left(\Gamma_{0}\right)$. Then the Jacobian $J_{f}=1$ and the norm of the derivative is $\left|\dot{c}_{x}\right|=\sqrt{1+\beta^{2}}$. Besides, $\ell=\left(1+\beta^{2}\right)^{q / 2} r$, $\rho_{0}=\frac{1}{r \sqrt{1+\beta^{2}}}$, and the $p$-module becomes

$$
M_{p}\left(\Gamma_{0}^{\prime}\right)=\frac{H^{n-1}(D)}{\left(1+\beta^{2}\right)^{p / 2} r^{p-1}} .
$$

At the same time, let us calculate the $q$-module in Theorem 4 under the same transform. Obviously, $\ell=\mu(D)$ and

$$
M_{q}\left(\Sigma_{0}^{\prime}\right)=\frac{r}{\left(H^{n-1}(D)\right)^{q-1}} .
$$

We see that $\left(M_{p}\left(\Gamma_{0}^{\prime}\right)\right)^{q}\left(M_{q}\left(\Sigma_{0}^{\prime}\right)\right)^{p} \neq 1$, and the function $\rho_{0}=\frac{1}{r \sqrt{1+\beta^{2}}}$ is no longer admissible for a larger family $\Gamma^{\prime}=f(\Gamma)$ while other conditions of Beurling-Badger's criterion applied to the families $\Gamma^{\prime}$ and $\Gamma_{0}^{\prime}$ hold. The statements on $\Sigma, \Sigma_{0}$ and $\Sigma^{\prime}, \Sigma_{0}^{\prime}$ remain as in Example 3 and in some cases can give interesting estimates as in Example 1. At the same time, using monotonicity of modules we can derive estimates for the module of the whole family of curves connecting $D_{0}$ and $D_{1}$ as

$$
\frac{H^{n-1}(D)}{\left(1+\beta^{2}\right)^{p / 2} r^{p-1}}=M_{p}\left(\Gamma_{0}^{\prime}\right) \leq M_{p}\left(\Gamma^{\prime}\right)=\left(M_{q}\left(\Sigma^{\prime}\right)\right)^{p / q} \leq\left(M_{q}\left(\Sigma_{0}^{\prime}\right)\right)^{p / q}=\frac{H^{n-1}(D)}{r^{p-1}} .
$$

Example 5. Let us first give known expressions for the $p$-module of the family of locally rectifiable curves $\Gamma$ connecting $S_{a}$ and $S_{b}$ in the spherical ring domain $R_{a b}$ bounded by $S_{a}$ and $S_{b}$. The function

$$
\rho_{0}(x)= \begin{cases}\left.\frac{|p-n|}{p-1}\left|b^{\frac{p-n}{p-1}}-a^{\frac{p-n}{p-1}}\right|^{-1}|\nabla| x\right|^{\frac{p-n}{p-1}} \mid, & \text { for } p \neq n, \\ (\log b / a)^{-1}|\nabla \log | x||, & \text { for } p=n\end{cases}
$$

is admissible for $\Gamma$ and $\int_{\gamma \in \Gamma_{0}} \rho_{0} d s=1$. Besides,

$$
M_{p}(\Gamma)=\int_{R_{a b}} \rho_{0}^{p} d y= \begin{cases}\left(\frac{|p-n|}{p-1}\right)^{p-1}\left|b^{\frac{p-n}{p-1}}-a^{\frac{p-n}{p-1}}\right|^{1-p} \omega\left(S_{1}\right), & \text { for } p \neq n \\ (\log b / a)^{1-n} \omega\left(S_{1}\right), & \text { for } p=n\end{cases}
$$

We will prove analogous formulas in details later for a more general case of polarizable groups, see also [29, 74, 88].

Let us consider a spherical ring $R_{1 r}$ bounded by the unit sphere $S_{1}$ and a sphere $S_{r}$ of radius $r, 1<r<\pi$. Recall that $R_{1 r}$ is given by the spherical transformation $G:\left(\theta_{1}, \ldots, \theta_{n-1}, t\right) \rightarrow\left(x_{1}, \ldots, x_{n}\right)$, where $\theta_{1} \in[0,2 \pi), \theta_{k} \in$ 
$[0, \pi], k=2,3, \ldots, n-1, t \in[1, r]$, and

$$
\begin{aligned}
& x_{1}=t \sin \theta_{1} \sin \theta_{2} \ldots \sin \theta_{n-1}, \\
& x_{2}=t \cos \theta_{1} \sin \theta_{2} \ldots \sin \theta_{n-1}, \\
& x_{3}=t \cos \theta_{2} \sin \theta_{3} \ldots \sin \theta_{n-1}, \\
& \ldots \quad \cdots \\
& x_{n}=t \cos \theta_{n-1} .
\end{aligned}
$$

The Jacobian $J_{G}$ of the spherical transformation $G$ is

$$
J_{G}\left(\theta_{1}, \ldots, \theta_{n-1}, t\right)=t^{n-1} \omega=t^{n-1} \sin \theta_{2} \sin ^{2} \theta_{3} \ldots \sin ^{n-2} \theta_{n-1} .
$$

The line element $d s$ is computed as

$$
\begin{aligned}
d s^{2}=d t^{2} & +t^{2}\left(d \theta_{n-1}^{2}+\sin ^{2} \theta_{n-1} d \theta_{n-2}^{2}+\sin ^{2} \theta_{n-1} \sin ^{2} \theta_{n-2} d \theta_{n-3}^{2}\right. \\
& \left.+\cdots+\sin ^{2} \theta_{n-1} \sin ^{2} \theta_{n-2} \ldots \sin ^{2} \theta_{2} d \theta_{1}^{2}\right) .
\end{aligned}
$$

Let us define the twisting map $f$ of $R_{1 r}$ by the shear transform

$$
G^{-1} \circ f \circ G:\left(\theta_{1}, \theta_{2}, \ldots, \theta_{n-1}, t\right) \rightarrow\left(\left(\theta_{1}+t-1\right), \theta_{2}, \ldots, \theta_{n-1}, t\right),
$$

i.e., the boundary sphere $S_{1}$ remains unchanged while the spheres $S_{t}$ rotate by an angle $t-1, t \in(1, r]$ by the element of $\mathrm{SO}(n)$

$$
\left(\begin{array}{rrrrrr}
\cos (t-1) & \sin (t-1) & 0 & 0 & \ldots & 0 \\
-\sin (t-1) & \cos (t-1) & 0 & 0 & \ldots & 0 \\
0 & 0 & 1 & 0 & \ldots & 0 \\
\ldots & \ldots & \ldots & \ddots & \ldots & \ldots \\
0 & 0 & 0 & 0 & \ldots & 1
\end{array}\right) .
$$

Observe that $J_{f}=J_{G^{-1} \circ f \circ G}=1$, and the radial intervals from $\Gamma_{0}$ are mapped onto the curves $c(t)$ with $|\dot{c}|=\sqrt{1+t^{2}}$. Theorem 3 implies that

$$
\ell(x)=\int_{1}^{r}\left(1+t^{2}\right)^{q / 2} I_{f}^{1-q} d t=\int_{1}^{r}\left(1+t^{2}\right)^{q / 2} t^{(n-1)(1-q)} d t=: K,
$$

and the $p$-module of $f\left(\Gamma_{0}\right)$ is

$$
M_{p}\left(f\left(\Gamma_{0}\right)\right)=\int_{S_{1}} \ell^{1-p} d x=K^{1-p} \int_{S_{1}} d x=K^{1-p} \omega\left(S_{1}\right) .
$$

Having in mind the previous example and the monotonicity of the module, we come to correct inequalities for the following integrals

$$
\int_{1}^{r}\left(1+t^{2}\right)^{\frac{p}{2(p-1)}} t^{\frac{n-1}{1-p}} d t \geq \frac{p-1}{|p-n|}\left(r^{\frac{p-n}{p-1}}-1\right), \quad p>1, \quad p \neq n,
$$

and

$$
\int_{1}^{r} \frac{\left(1+t^{2}\right)^{\frac{n}{2(n-1)}}}{t} d t \geq \log r, \quad p=n \geq 2
$$


Analogously we can define an automorphism $f$ of $R_{1 r}$ by the twisting map

$$
G^{-1} \circ f \circ G:\left(\theta_{1}, \theta_{2}, \ldots, \theta_{n-1}, t\right) \rightarrow\left(\left(\theta_{1}+\beta \log t\right), \theta_{2}, \ldots, \theta_{n-1}, t\right),
$$

i.e., the boundary sphere $S_{1}$ remains unchanged while the spheres $S_{t}$ rotate by an angle $\beta \log t, t \in(1, r]$. Then the radial intervals from $\Gamma_{0}$ are mapped onto the curves $c(t)$ with $|\dot{c}|=\sqrt{1+\beta^{2}}=$ const, and

$$
\ell(x) \equiv \begin{cases}\left(1+\beta^{2}\right)^{\frac{q}{2}} \frac{r^{\frac{p-n}{p-1}}-1}{p-n}(p-1), & \text { for } p \neq n, \\ \left(1+\beta^{2}\right)^{\frac{n}{2(n-1)}} \log r, & \text { if } q=\frac{n}{n-1} \text { and } p=n .\end{cases}
$$

Hence,

$M_{p}\left(f\left(\Gamma_{0}\right)\right)= \begin{cases}\frac{1}{\left(1+\beta^{2}\right)^{p / 2}}\left(\frac{|p-n|}{p-1}\right)^{p-1}\left|r^{\frac{p-n}{p-1}}-1\right|^{1-p} \omega\left(S_{1}\right), & \text { for } p \neq n, \\ \frac{\omega\left(S_{1}\right)}{\left(1+\beta^{2}\right)^{n / 2}(\log r)^{n-1}}, & \text { if } q=\frac{n}{n-1} \text { and } p=n .\end{cases}$

In particular, if $p=2$ and $n=2$, then $M_{2}\left(f\left(\Gamma_{0}\right)\right)=\frac{2 \pi}{\left(1+\beta^{2}\right) \log r}$. This, naturally, agrees with (12).

\subsection{Proof of Theorem 3 and 4 .}

\subsubsection{Proof of Theorem 3.}

Proof. Let us first observe that $\int_{c_{x}} \rho_{0} d s=1$. Indeed,

$$
\begin{aligned}
\int_{c_{x}} \rho_{0} d s & =\int_{a}^{b}\left(\rho_{0} \circ f \circ \mathbf{u}\right)\left|\dot{c}_{x}\right| d t=\frac{1}{\ell} \int_{a}^{b}\left(\frac{\left|\dot{c}_{x}\right|}{I_{f}}\right)^{\frac{1}{p-1}}\left|\dot{c}_{x}\right| d t \\
& =\frac{1}{\ell} \int_{a}^{b}\left(\frac{\left|\dot{c}_{x}\right|}{I_{f}}\right)^{q} I_{f} d t=1, \quad \text { for all } \quad x \in D .
\end{aligned}
$$

Therefore, $\rho_{0}$ is admissible for $f\left(\Gamma_{0}\right)$ and

$$
M_{p}\left(f\left(\Gamma_{0}\right)\right) \leq \int_{\Omega^{\prime}} \rho_{0}^{p} d y
$$

On the other hand, for any $\rho$ admissible for $f\left(\Gamma_{0}\right)$, we have $\int_{c_{x}} \rho d s \geq 1$, and therefore

$$
\int_{c_{x}}\left(\rho-\rho_{0}\right) d s \geq 0
$$

This implies that

$$
\frac{1}{\ell^{p-1}(x)} \int_{a}^{b}\left[\left(\rho-\rho_{0}\right) \circ f \circ \mathbf{u}\right]\left|\dot{c}_{x}\right| d t \geq 0 .
$$

Then

$$
\int_{D} \int_{a}^{b}\left(\left(\rho-\rho_{0}\right) \rho_{0}^{p-1} \circ f \circ \mathbf{u}\right) I_{f} d t d H_{x}^{n-1} \geq 0
$$


Equivalently,

$$
\int_{\Omega^{\prime}} \rho \rho_{0}^{p-1} d y \geq \int_{\Omega^{\prime}} \rho_{0}^{p} d y
$$

Thus we can apply the Remark 3, to conclude that $\rho_{0}$ is extremal for the module problem in consideration and that $M_{p}\left(f\left(\Gamma_{0}\right)\right)=\int_{\Omega^{\prime}} \rho_{0}^{p} d y$. Now we can calculate the $p$-module as

$$
\begin{aligned}
M_{p}\left(f\left(\Gamma_{0}\right)\right) & =\int_{\Omega^{\prime}} \rho_{0}^{p} d y=\int_{D} \int_{a}^{b}\left[\rho_{0}^{p} \circ f \circ \mathbf{u}\right] I_{f} d t d H_{x}^{n-1} \\
& =\int_{D} \int_{a}^{b} \frac{1}{\ell^{p}}\left(\frac{\left|\dot{c}_{x}\right|}{I_{f}}\right)^{\frac{p}{p-1}} I_{f} d t d H_{x}^{n-1}=\int_{D} \ell^{1-p} d H_{x}^{n-1} .
\end{aligned}
$$

The condition of regularity of the mapping $f$ required for Theorem 3 can be relaxed, so that $f$ belongs to some more general known classes of maps, see Section 3.4.4. For example, we can assume that $f$ is homeomorphic $W^{1,1}$ Sobolev and orientation preserving in $\mathbb{R}^{3}$, see [38], or quasiconformal in $\mathbb{R}^{n}$, see [86].

3.4.2. Coarea formula and change of variables. In order to prove Theorem 4 we need a variant of the coarea and change-of-variable formulas which we formulate first for smooth maps.

Let $u: \mathbb{R}^{n} \rightarrow \mathbb{R}$ be $C^{1}$-smooth, $f: \mathbb{R}^{n} \rightarrow \mathbb{R}^{n}$ a $C^{1}$ orientation preserving homeomorphism. Let $D$ be $H^{n-1}$-measurable set, $\Omega=D \times(-\infty, \infty)$, and $\Omega^{\prime}=f(\Omega)$. Let $\sigma_{r}=\{x \in \Omega:(u \circ f)(x)=r\}$ and $\sigma_{r}^{\prime}=\left\{y \in \Omega^{\prime}: u(y)=r\right\}$ be the level sets of $u \circ f$ and $u$ for a fixed $r$, respectively. A variant of Sard's theorem, see [21], states that $H_{y}^{n-1}\left(\sigma_{r}^{\prime} \cap Z_{u}\right)=0$ for almost all $r \in \mathbb{R}$, where $Z_{u}$ is the set of the points where the Jacobian $J_{u}$ vanishes.

In particular, let us consider the condenser $\left(\Omega ; D_{0}, D_{1}\right)$ in $\mathbb{R}^{n}$ parametrised by an embedding $(x, t) \mapsto \mathbf{u}(x, t)$, where $x \in D \subset \mathbb{R}^{n}, D$ is $(n-1)$-Hausdorff dimensional compact , $\mathbf{u}=\left(u_{1}, \ldots, u_{n}\right)$ is $C^{1}$-smooth homeomorphism with positive Jacobian $J_{\mathbf{u}}$, and $t \in[a, b]$. The function $u$ will be defined to be $u(y)=t \circ f^{-1}(y)$ where $t(\cdot)$ is the last coordinate $t$ of $(x, t)$ of the inverse map $\mathbf{u}^{-1}$. Define $I_{f}=J_{f} J_{\mathbf{u}}$.

Let us recall the change of variables formula. For any Borel set $U \subset \mathbb{R}^{n}$ and for any homeomorphism $f: \mathbb{R}^{n} \rightarrow \mathbb{R}^{n}$ we define the pull-back of the Hausdorff measure $\mu$ by $f$ as $f^{*} \mu(U) \equiv(\mu \circ f)(U)=\mu(f(U))$. Then $\mu \circ f$ is absolutely continuous with respect to $\mu$ and the Radon-Nikodym theorem implies the change of variables formula

$$
\int_{f(U)} \rho^{\prime} d \mu=\int_{U}\left(\rho^{\prime} \circ f\right) \frac{d(\mu \circ f)}{d \mu} d \mu .
$$


Lemma 1. For a positive measurable function $\rho$ in $\mathbb{R}^{n}$ the following change of variables formula holds

$$
\int_{\sigma_{r}^{\prime}} \rho d H^{n-1}(y)=\int_{\sigma_{r}} \frac{\rho|\nabla u| \circ f}{|\nabla(u \circ f)|} J_{f} d H^{n-1}(x) .
$$

In addition, if $u=t \circ f^{-1}(y)$, then

$$
\int_{\sigma_{r}^{\prime}} \rho d H^{n-1}(y)=\int_{\sigma_{r}}\left(\rho \mid\left(\nabla\left(t\left(f^{-1}\right)\right) \circ f(\mathbf{u}(x, t)) \mid\right) I_{f} d H^{n-1}(x) .\right.
$$

Proof. Indeed, the conditions on the function $u$ and the mapping $f$ ensure that both $u$ and $u \circ f$ are $C^{1}$-smooth. For a fixed $r$ denote by $B_{r}=\{x \in$ $\Omega: u \circ f(x) \leq r\}$ and $B_{r}^{\prime}=\left\{y \in \Omega^{\prime}: u(y) \leq r\right\}$.

By the co-area formula (see, e.g., [53]) we have that

$$
\int_{B_{r}^{\prime}} \rho|\nabla u| d y=\int_{-\infty}^{r} \int_{\sigma_{r}^{\prime}} \rho d H^{n-1}(y) d r .
$$

Then

$$
\frac{\partial}{\partial r} \int_{B_{r}^{\prime}} \rho|\nabla u| d y=\int_{\sigma_{r}^{\prime}} \rho d H^{n-1}(y)
$$

holds as the above derivative exists for almost all $r$, see, e.g., [60, pp. 29-33].

By the change of variables formula (see [33])

$$
\int_{B_{r}^{\prime}} \rho|\nabla u| d y=\int_{B_{r}}(\rho|\nabla u|) \circ f J_{f} d x .
$$

Applying the co-area formula, once more and in a similar fashion as before, to the right hand side of the above equation and differentiating with respect to $r$ gives

$$
\frac{\partial}{\partial r} \int_{B_{r}}(\rho|\nabla u|) \circ f J_{f} d x=\int_{\sigma_{r}} \frac{(\rho|\nabla u|) \circ f}{|\nabla(u \circ f)|} J_{f} d H^{n-1}(x) .
$$

Comparison of the right-hand sides in (16) and (17) leads to the first statement of Lemma 1 .

To prove the second part of Lemma 1, observe that we set $u=t\left(f^{-1}\right)$. Thus $\sigma_{r}=\left\{x:\left(x_{1}, \ldots, x_{n-1}\right) \in D, t=r \in(a, b)\right\}$ and $\sigma_{r}^{\prime}$ is a level set of the function $t\left(f^{-1}\right)$, i.e., $\sigma_{r}^{\prime}=\left\{y \in f(\Omega): t\left(f^{-1}\right)=r\right\}$. Then we must change $f \rightarrow f \circ \mathbf{u}$ and $u \rightarrow t\left(f^{-1}\right)$ in (14). The gradient in the denominator $|\nabla(u \circ f)|$ in (14) becomes $\left|\nabla\left(t\left(f^{-1}\right) \circ f \circ \mathbf{u}\right)\right|=|(0, \ldots, 1)|=1$ from where follows (15).

Lemma 1 implies that in our case the Radon-Nikodym derivative becomes

$$
\frac{d(\mu \circ f)}{d \mu}=\mid\left(\nabla\left(t\left(f^{-1}\right)\right) \circ f(\mathbf{u}(x, t)) \mid I_{f}(x) .\right.
$$




\subsubsection{Proof of Theorem 目.}

Proof. The proof is similar to that of Theorem 3 where we substitute RadonNikodym derivative (18) in place of $\left|\dot{c}_{x}\right|$ in the claim on admissibility of $\rho_{0}$, and use Lemma 1 when the change-of-variable formula (15) is applied. First we show that $\rho_{0}$ is an admissible function:

$$
\begin{gathered}
\int_{\sigma_{t}^{\prime}} \rho_{0} d H^{n-1}=\int_{\sigma_{t}^{\prime}} \frac{1}{\ell\left(t\left(f^{-1}\right)\right)}\left(\left|\nabla t\left(f^{-1}\right)\right|\right)^{1 /(q-1)} d H^{n-1}= \\
\int_{\sigma_{t}} \frac{1}{\ell\left(t\left(f^{-1}\right)\right)}\left(\left|\nabla t\left(f^{-1}\right)\right|\right)^{1 /(q-1)}\left|\nabla t\left(f^{-1}\right)\right| I_{f} d H^{n-1}= \\
\frac{1}{\ell\left(t\left(f^{-1}\right)\right)} \int_{\sigma_{t}}\left|\nabla t\left(f^{-1}\right)\right|^{p} I_{f} d H^{n-1}=1 .
\end{gathered}
$$

Now let $\rho$ be any admissible function for $E_{0}^{\prime}$, i.e. $\int_{\sigma_{t}^{\prime}} \rho d H^{n-1} \geq 1$. Since $\int_{\sigma_{t}^{\prime}} \rho_{0} d H^{n-1} \geq 1$, we have

$$
\frac{1}{\ell^{q-1}} \int_{\sigma_{t}^{\prime}}\left(\rho-\rho_{0}\right) d H^{n-1} \geq 0
$$

and by Lemma 1

$$
\frac{1}{\ell^{q-1}} \int_{\sigma_{t}}\left(\left(\rho-\rho_{0}\right)\left|\nabla t\left(f^{-1}\right)\right|\right) \circ f I_{f} d H^{n-1} \geq 0 .
$$

Since $\left|\nabla t\left(f^{-1}\right)\right|=\ell^{q-1} \rho^{q-1} \circ f \circ \mathbf{u}$, it follows that

$$
\int_{a}^{b} \int_{\sigma_{t}}\left(\left(\rho-\rho_{0}\right) \rho_{0}^{q-1}\right) \circ f \circ \mathbf{u} I_{f} d H^{n-1} d t \geq 0 .
$$

By Fubini's theorem and change of variables formula we obtain that $\int_{\Omega^{\prime}}(\rho-$ $\left.\rho_{0}\right) \rho_{0}^{q-1} d y \geq 0$, and therefore applying Remark 3 , we conclude that $\rho_{0}$ is extremal for the module problem under consideration and that $M_{q}\left(\Sigma_{0}^{\prime}\right)=$ $\int_{\Omega^{\prime}} \rho_{0}^{q} d y$.

3.4.4. Theorem 3 and 4 for non-smooth homeomorphisms. The conditions we imposed on the function $u$ and the map $f$ can be relaxed. Let us analyse the following ingredients of the above lemmas. We need the following properties:

(i) The function $u: \mathbb{R}^{n} \rightarrow \mathbb{R}$ and the homeomorphism $f: \mathbb{R}^{n} \rightarrow \mathbb{R}^{n}$ must be such that $u$ and the superposition $u \circ f$ are of the same type of regularity;

(ii) The homeomorphism $f: \mathbb{R}^{n} \rightarrow \mathbb{R}^{n}$ and its inverse $f^{-1}$ must be of the same type of regularity;

(iii) The critical set for the level sets $\sigma_{r}$ and $\sigma_{r}^{\prime}$ must have the $H^{n-1}$-measure zero.

First, let us describe the level sets of the function $u$. 
Definition 6. Given integers $1 \leq k \leq n$, we say that a Borel set $B \subset \mathbb{R}^{n}$ is countably Hausdorff $H^{k}$-rectifiable if there exists a sequence of Lipschitz maps $\psi_{i}: E_{i} \subset \mathbb{R}^{k} \rightarrow \mathbb{R}^{n}$ such that $H^{k}\left(B \backslash \bigcup_{i=1}^{\infty} \psi_{i}\left(E_{i}\right)\right)=0$.

The set $B \subset \mathbb{R}^{n}$ is countably $H^{k}$-rectifiable if and only if there exists a sequence of $k$-dimensional $C^{1}$-smooth manifolds $M_{1}, M_{2}, \ldots$, such that $H^{k}\left(B \backslash \bigcup_{i=1}^{\infty} M_{i}\right)=0$, see [22, 34]. A version of Sard's theorem tells us that if $u: \mathbb{R}^{n} \rightarrow \mathbb{R}$ is Lipschitz, then for almost every point $r \in \mathbb{R}, u^{-1}(r)$ is countably $H^{n-1}$-rectifiable, see [34]. If $u \in W^{1, p}\left(\mathbb{R}^{n}, \mathbb{R}\right)$, then there exists a Borel representative of $u$ such that $u^{-1}(r)$ is countably $H^{n-1}$-rectifiable for almost all $r$, see [5, 9, 22, 34, 53] for more on rectifiable sets. We remark that this analogue of Sard's theorem is not enough to state the coarea formula. The following definition is found in [67].

Definition 7. A function $u: \mathbb{R}^{n} \rightarrow \mathbb{R}$ is called p-quasicontinuous if it is Borel measurable and continuous on a set $\mathbb{R}^{n} \backslash U$ where $U$ is a set of arbitrary small p-capacity.

Reshetnyak's theorem [67] claims that any $W^{1, p}\left(\mathbb{R}^{n}, \mathbb{R}\right)$ Sobolev function, $p \geq 1$, has a $p$-quasicontinuous representative for $1 \leq p \leq n$, or a continuous representative for $p>n$. Now let $u: \mathbb{R}^{n} \rightarrow \mathbb{R}$ be a $p$-quasicontinuous representative of a $W^{1, p}$-Sobolev function, $p \geq 1$, and let $g: \mathbb{R}^{n} \rightarrow \mathbb{R}$ be positive and measurable. Then the coarea formula (16) holds, where $\nabla$ means the distributional gradient.

We discuss now the composition operators. We say that a locally Lipschitz function $u: \mathbb{R}^{n} \rightarrow \mathbb{R}$ satisfies the polynomial growth condition for its partial derivatives almost everywhere in $\mathbb{R}^{n}$, if

$$
\left|\frac{\partial u}{\partial x_{j}}(x)\right| \leq a\left(1+\left|x_{j}\right|^{\nu}\right), \quad \text { a.e. in } \mathbb{R}^{n}, j=1, \ldots, n,
$$

where $\nu=\frac{n(p-r)}{r(n-p)}, \quad 1 \leq r \leq p<n<\infty$, and $a>0$ is a constant. Given a function $u: \mathbb{R}^{n} \rightarrow \mathbb{R}$, and a map $f \in W^{1, p}\left(\Omega, \mathbb{R}^{n}\right), p \geq 1$ let us define a composition operator $T_{u}$ by $T_{u}(f)=u \circ f$. The operator $T_{u}$ maps $W^{1, p}\left(\Omega, \mathbb{R}^{n}\right)$ into $W^{1, r}(\Omega, \mathbb{R})$, if and only if, $1 \leq p<n$, the domain $\Omega$ is bounded domain in $\mathbb{R}^{n}$ satisfying the cone condition, and $u$ is locally Lipschitz having its partial derivatives of polynomial growth, see Marcus and Mizel [58]. For $p>n$, the polynomial growth of the partial derivatives can be omitted.

A different composition operator $T_{f}: W^{1, p}\left(\Omega^{\prime}, \mathbb{R}\right) \rightarrow W^{1, p}(\Omega, \mathbb{R})$ defined by $T_{f}(u)=u \circ f$ was considered in a series of papers [30, 79, 80]. It was proved that the necessary and sufficient condition for $T_{f}$ to induce an isomorphism of $W^{1, p}$ spaces is that $f$ is quasiisometric. Ukhlov and Vodopyanov [82, 83] studied also the operator $T_{f}: L^{1, p}\left(\Omega^{\prime}, \mathbb{R}\right) \rightarrow L^{1, q}(\Omega, \mathbb{R})$, for Sobolev spaces whose Sobolev norm does not contain $\|u\|_{p}$. A necessary and sufficient condition involved 
ACL function of bounded distortion, which in particular, included the case $p=n$. Let us remark that $A C L^{p}=W^{1, p}$ in the sense that any $A C L^{p}$ function represents a function from $W^{1, p}$ and any $W^{1, p}$ function contains an $A C L^{p}$ representative.

Finally, let us turn to the problem of regularity of the inverse map. For this we need the definition of a function of finite distortion, see [40].

Definition 8. A map $f: \Omega \rightarrow \mathbb{R}^{n}$ on an open set $\Omega \subset \mathbb{R}^{n}$ has finite distortion if $f \in W_{\text {loc }}^{1,1}\left(\Omega, \mathbb{R}^{n}\right)$, the Jacobian $J_{f} \geq 0$ almost everywhere in $\Omega, J_{f} \in L_{\text {loc }}^{1}(\Omega)$ and there exists some function $K: \Omega \rightarrow[1, \infty]$ finite almost everywhere in $\Omega$ such that

$$
\|D f(x)\|^{n} \leq K(x) J_{f}(x), \quad \text { a.e. in } \Omega \text {. }
$$

Let $\Omega$ be an open set in $\mathbb{R}^{n}$, and let $f \in W^{1, n-1}\left(\Omega, \mathbb{R}^{n}\right)$ be a homeomorphism of finite distortion, $\Omega^{\prime}=f(\Omega)$. A result by Csörnyei, Hencl, and Malý [19] implies that $f^{-1} \in W^{1, n-1}\left(\Omega^{\prime}, \mathbb{R}^{n}\right)$ and $\left(f^{-1}\right)_{n} \in W^{1, n-1}\left(\Omega^{\prime}, \mathbb{R}\right)$. For the results on the functions of the Sobolev class $L^{1, p}$, see [84].

Conclusion. In view of the above, the conditions on the set $\Omega \subset \mathbb{R}^{n}$, on the function $u$, and on the mapping $f$ in Lemma 1 can be chosen, for example, as follows.

- The function u can be chosen to be locally Lipschitz function from $W^{1, p}$ satisfying the polynomial growth condition for its partial derivatives for $1 \leq p<n$, and the homeomorphism $f$ to be a p-quasicontinuous representative of a $W^{1, p}$-Sobolev map in Lemma 1;

- The homeomorphism $f$ in Lemma 1 can be chosen to be $W^{1, n-1}$-Sobolev of finite distortion.

\section{Extremal measures on polarizable groups}

In this section we want to prove an analogue of Rodin's theorem and to discuss extremal functions and extremal families for the module of curves and separating sets in a geometry different from the Euclidean one, namely for a special type of Carnot groups. We start with some necessary definitions.

\subsection{Definition of polarizable groups.}

Definition 9. The Carnot group $G$ is a connected, simply connected Lie group, whose Lie algebra $\mathfrak{g}$ is nilpotent and possesses a stratification $\mathfrak{g}=\bigoplus_{j=1}^{l} V_{j}$, where $\left[V_{1}, V_{j}\right]=V_{j+1}$ for all $j \in \mathbb{N}$ with $V_{j}=\{0\}$, whenever $j>l$. The positive integer $l$ is called a step of the group.

We assume that the underlying layer $V_{1}$ is endowed with an inner product $\langle., .\rangle_{0}$, and let $X_{1}, \cdots, X_{k}$ be an orthonormal basis of $V_{1}$ with respect to this inner product. The vector fields $X_{1}, \cdots, X_{k}$ are usually called horizontal, and 
a sub-bundle $H G$ of the tangent bundle $T G$ of the group $G$ with the typical fiber $H_{g} G=\operatorname{span}\left\{X_{1}(g), \cdots, X_{k}(g)\right\} \subset T_{g} G, g \in G$, is called a horizontal sub-bundle. As a consequence, any vector $v \in H_{g} G$ is also called horizontal. The inner product $\langle., .\rangle_{0}$ on $V_{1}$ defines a left-invariant sub-Riemanian metric on $G$ through the left translations, which we denote by the same symbol. We write $\|v\|_{0}^{2}=\langle v, v\rangle_{0}$ for $v \in H_{g} G$. Let us use the normal coordinates of the first kind, where an element $g \in G$ is identified with $\left(x_{1}, \cdots, x_{k}, t_{k+1}, \cdots, t_{m}\right) \in \mathbb{R}^{m}$ by the formula

$$
g=\exp \left(\sum_{i=1}^{k} x_{i} X_{i}+\sum_{i=k+1}^{m} t_{i} T_{i}\right)
$$

where $T_{k+1}, \cdots, T_{m}$ denotes a set of vectors, extending the horizontal basis $X_{1}, \cdots, X_{k}$ to the entire basis of $\mathfrak{g}$. The stratified structure of the Lie algebra naturally defines the dilation $\delta_{s}, s>0$, that in the introduced coordinates can be written as

$$
\begin{aligned}
\delta_{s} g & =\delta_{s}\left(x_{1}, \cdots, x_{k}, t_{k+1}, \cdots, t_{k+\operatorname{dim}\left(V_{2}\right)}, \ldots, t_{k+\sum_{j=1}^{l-1} \operatorname{dim}\left(V_{j}\right)+1}, \cdots, t_{m}\right) \\
& =\left(s x_{1}, \cdots, s x_{k}, s^{2} t_{k+1}, \cdots, s^{2} t_{k+\operatorname{dim}\left(V_{2}\right)}, \ldots, s^{l} t_{k+\sum_{j=1}^{l-1} \operatorname{dim}\left(V_{j}\right)+1}, \cdots, s^{l} t_{m}\right) .
\end{aligned}
$$

As a simply connected nilpotent group, admitting dilations $\delta_{s}, G$ is globally diffeomorphic to $\mathfrak{g} \cong \mathbb{R}^{m}, m=\sum_{i=1}^{l} \operatorname{dim} V_{i}$, via the exponential map, see [23, Proposition 1.2]. The number $m$ is called a topological dimension of the group. The sub-Riemannian metric induces the distance function $d_{c c}$ on $G$ in the same way as it does for a Riemannian metric. The distance function $d_{c c}$ is usually called the Carnot-Carathéodory distance, and the Hausdorff dimension of the metric space $\left(G, d_{c c}\right)$ is equal to $Q=\sum_{i=1}^{l} i \operatorname{dim} V_{i}$, see [59]. The number $Q$ is also called a homogeneous dimension of $G$, and it will play an important role in the forthcoming calculations. The Haar measure on $G$ is induced by the exponential map from the Lebesgue measure on $\mathfrak{g} \cong \mathbb{R}^{m}$. A norm $N_{G}$ on the group $G$ is called homogeneous if it is a homogeneous of order one function with respect to the dilation $\delta_{s}: N_{G}\left(\delta_{s} g\right)=s N_{G}(g)$ for all $g \in G$.

The horizontal gradient $\nabla_{0}$ is a unique horizontal vector such that

$$
\left\langle\nabla_{0} f, v\right\rangle_{0}=v(f), \quad \text { for any } \quad v \in H_{g} G, \quad f \in C^{\infty}(G) .
$$

The horizontal gradient is expressed in the orthonormal basis $X_{1}, \ldots, X_{k}$ as $\nabla_{0} f=\left(X_{1} f, \ldots, X_{k} f\right)$.

Given a domain $U \subset G$, a function $u \in C^{2}(U)$ is called $p$-harmonic if it satisfies the $p$-sub-Laplace equation in $U$ :

$$
\Delta_{0, p} u:=\sum_{i=1}^{k} X_{i}\left(\left\|\nabla_{0} u\right\|_{0}^{p-2} X_{i} u\right)=0
$$


and $\infty$-harmonic if it satisfies the $\infty$-sub-Laplace equation in $U$ :

$$
\Delta_{0, \infty} u:=\frac{1}{2}\left\langle\nabla_{0}\left\|\nabla_{0} u\right\|_{0}^{2}, \nabla_{0} u\right\rangle_{0}=0 .
$$

The derivatives of $u$ can also be understood in the generalised sense. By a result of Folland [24, Theorem 2.1], there exists a unique fundamental solution $u_{2}$ in any Carnot group $G$ to the Kohn sub-Laplacian $\Delta_{0,2}$, which is smooth away from zero and homogeneous of degree $2-Q: u_{2} \circ \delta_{s}=s^{2-Q} u_{2}$.

Definition 10. [7] We say that a Carnot group $G$ is polarizable if the fundamental solution $u_{2}$ of the Kohn sub-Laplacian $\Delta_{0,2}$ has the property that the homogeneous norm $N_{G}=u_{2}^{\frac{1}{2-Q}}$ associated with $u_{2}$ is $\infty$-harmonic away from zero in $G$.

Examples of polarizable groups are $\mathbb{R}^{m}, n$-th Heisenberg group $\mathbb{H}^{n}$, and $H$ (eisenberg)-type groups introduced by Kaplan [43], which definition will be given in Section 4.1.2. The main result of [7] is that in any polarazible group it is possible to carry out the construction of some sort of spherical coordinates in the same way as it has been done in [47] for the Heisenberg group.

Let $G$ be a polarizable Carnot group, and let $N_{G}$ be a norm from Definition 10. We denote by $\mathcal{Z}$ the characteristic set of the function $N_{G}$ :

$$
\mathcal{Z}:=\{0\} \cup\left\{g \in G \backslash\{0\} \mid \nabla_{0} N_{G}(g)=0\right\} .
$$

The radial flow is the solution to the Cauchy initial-value problem in $G \backslash \mathcal{Z}$

$$
\left\{\begin{array}{l}
\frac{\partial}{\partial s} \phi(s, g)=\frac{N_{G}(\phi(s, g))}{s} \cdot \frac{\nabla_{0} N_{G}(\phi(s, g))}{\left\|\nabla_{0} N_{G}(\phi(s, g))\right\|_{0}^{2}}, \\
\phi(1, g)=g .
\end{array}\right.
$$

Proposition 7. 7] The flow $\phi$ satisfies the following properties:

(i) $N_{G}(\phi(s, g))=s N_{G}(g)$ for $s>0$, and $g \in G \backslash \mathcal{Z}$;

(ii) $\|(\partial \phi / \partial s)\|_{0}$ is independent of $s$, i.e.,

$$
\|(\partial \phi / \partial s)\|_{0}=\frac{N_{G}(g)}{\left\|\nabla_{0} N_{G}(\phi(s, g))\right\|_{0}}=: \lambda(g)^{-1}
$$

for a non-zero real-valued function $\lambda$ on $G \backslash \mathcal{Z}$;

(iii) $\operatorname{det} D_{g} \phi(s, g)=s^{Q}$ for $s>0$ and $g \in G \backslash \mathcal{Z}$, where $D_{g} \phi$ denotes the differential of the map $\phi(s, \cdot): G \backslash \mathcal{Z} \rightarrow G \backslash \mathcal{Z}$, where $G \backslash \mathcal{Z}$ is considered as a domain in $\mathbb{R}^{m}$.

Definition 11. An absolutely continuous curve $c: I \rightarrow G$ is called horizontal if the vector $\frac{d}{d s} c(s)$ is horizontal, i.e., $\frac{d}{d s} c(s) \in H_{c(s)} G$ for all $s \in I$, when it is defined. 
The solution $\phi$ of the Cauchy problem (20) is a horizontal curve simply because its tangent vector $\frac{\partial \phi(s, g)}{\partial s}$ is proportional to the horizontal gradient of some function, namely, of the homogeneous norm.

At the end of this section we notice that if $c: I \rightarrow G$ is a horizontal curve whose locus belongs to the level set of the function $N_{G}$, then

$$
\frac{d}{d \tau} N_{G}(c(\tau))=\left\langle\nabla_{0} N_{G}, \frac{d c(\tau)}{d \tau}\right\rangle_{0}=0 .
$$

We say that the horizontal gradient is orthogonal to the level set meaning that it is orthogonal to any horizontal curve (whose locus belongs to the level set) with respect to the inner product $\langle., .\rangle_{0}$. As a consequence, we conclude that the flow $\phi$ solving Cauchy problem (20) is orthogonal to the level set of the function $N_{G}$, where the orthogonality is understood with respect to the inner product $\langle., .\rangle_{0}$ of the tangent vector to the flow and the tangent vectors to the horizontal curves lying on the level set.

Note that not all Carnot groups are polarizable. It is known that the fundamental solution to 2-sub-Laplacian always exists, but it is not necessarily $\infty$-harmonic. An example of such kind of groups can be anisotropic $H$-type groups, see reasoning in [7]. Anisotropic $H$-type groups were studied for instance in [16, 18]. Now we continue with examples of polarizable groups.

4.1.1. Euclidean space. First of all, notice that $G=\mathbb{R}^{k}$ is a Carnot group of step 1 , where the Lie algebra is the space $V_{1}=\operatorname{span}\left\{X_{1}, \ldots, X_{k}\right\}$ with $X_{j}=$ $\frac{\partial}{\partial x_{j}}$. Since the commutation relations of $X_{j}, j=1, \ldots, k$ all vanish, the spaces $V_{2}=\ldots=V_{l}=\{0\}$. The exponential map is a map identifying $\mathbb{R}^{k}$ with $V^{1}$. The Kohn sub-Laplacian $\Delta_{0,2}$ is the usual Laplacian $\Delta_{2}$, whose fundamental solution $u_{2}(x)=|x|^{2-n}$ defines the homogeneous norm $N_{\mathbb{R}^{k}}(x)=|x|$, which is the Euclidean norm of the element $x \in \mathbb{R}^{k}$. It is trivial to check that $|\cdot|$ is an $\infty$-harmonic function. The radial flow $\phi(s, x)=s x, x \in \mathbb{R}^{k}$ is the solution to the corresponding Cauchy problem (20).

\subsubsection{H-type groups.}

Definition 12. We say that a Carnot group $G$ is of Heisenberg type (H-type) if its Lie algebra $\mathfrak{g}=V_{1} \oplus V_{2}$ of $G$ is 2 step, and if it is endowed with an inner product $\langle.,$.$\rangle , and admits a linear map J: V_{2} \rightarrow \operatorname{End}\left(V_{1}\right)$, compatible with the inner product in the following sense:

(1) $\left\langle J_{Z} U, V\right\rangle=\langle Z,[U, V]\rangle$ for all $Z \in V_{2} \quad U, V \in V_{1}$ and

(2) $J_{Z}^{2}=-\|Z\| \mathrm{Id} \quad$ for all $Z \in V_{2}$, where $\|Z\|^{2}=\langle Z, Z\rangle$.

The inner product $\langle., .\rangle_{0}$ on $V_{1}$ is the restriction of $\langle.,$.$\rangle on V_{1}$.

Let $G$ be a group of $H$-type. Since the exponential map of $G$ is an analytic diffeomorphism, we can define real analytic mappings $u: G \rightarrow V_{1}$ and $z: G \rightarrow$ 
$V_{2}$ by $g=\exp (u(g)+z(g)), g \in G$. The function

$$
N_{G}(g)=\left(\|u(g)\|^{4}+16\|z(g)\|^{2}\right)^{1 / 4}
$$

is a homogeneous norm on $G$. It is well known that $N_{G}$ is smooth on $G \backslash\{0\}$, see [43, Theorem 2]. It was shown in [7, Proposition 5.6] that $H$-type groups are polarizable with the norm $N_{G}$, defined in (21).

4.1.3. Heisenberg group. An example of $H$-type groups is the Heisenberg group.

Definition 13. The n-dimensional Heisenberg group $\mathbb{H}^{n}$ is an analytic, nilpotent Lie group whose underlying manifold is $\mathbb{R}^{2 n+1}$, and whose Lie algebra $\mathfrak{h}$ is graded

(1) $\mathfrak{h}=V_{1} \oplus V_{2}$, where $\operatorname{dim}\left(V_{1}\right)=2 n$ and $\operatorname{dim}\left(V_{2}\right)=1$, and

(2) $\mathfrak{h}$ admits the following commutation relations:

$$
\left[V_{1}, V_{1}\right]=V_{2}, \quad\left[V_{1}, V_{2}\right]=\left[V_{2}, V_{2}\right]=\{0\} .
$$

Choose any inner product $\langle.,$.$\rangle on \mathfrak{h}$, and define the map $J: V_{2} \rightarrow \operatorname{End}\left(V_{1}\right)$ as follows. Given any $U \in V_{1}$, define $\operatorname{ad}_{U}: V_{1} \rightarrow V_{2}$ by $\operatorname{ad}_{U} V:=[U, V]$. Then the map $J$ is the formal adjoint map $J=\operatorname{ad}_{U}^{*}$ given by $\left\langle J_{Z} U, V\right\rangle=\left\langle Z, \operatorname{ad}_{U} V\right\rangle$.

Using normal coordinates of the first kind $g=(x, y, t), x, y \in \mathbb{R}^{n}, t \in \mathbb{R}$, we write the homogeneous norm, for instance, as

$$
N_{G}(x, y, t)=\left(\left(|x|^{2}+|y|^{2}\right)^{2}+16 t^{2}\right)^{1 / 4} \text {. }
$$

4.2. The $p$-module of $\Gamma\left(R_{a b} ; S_{a}, S_{b}\right)$ on polarizable groups. Let $B(g, r)$, $g \in G$, be an open ball with respect to the norm $N_{G}$ in a polarizable Carnot group $G$, and let $S_{r}=\partial B(g, r)$ be the boundary of $B(g, r)$. We want to present the extremal function and the extremal family of curves for the condenser $\left(R_{a b} ; S_{a}, S_{b}\right)$ in the problem of the $p$-module.

One of the results in [47] by Korányi and Reimann is the precise value of $M_{p}\left(\Gamma\left(\left(R_{a b} ; S_{a}, S_{b}\right)\right)\right)$ in $\mathbb{H}^{1}$, where $p=4$ is the homogeneous dimension of $\mathbb{H}^{1}$. The $p$-module of the family of curves for the spherical ring domain on $H$ type groups and polarizable Carnot groups, in terms of $p$-capacity is given in [7, 17]. It is known, that the value of $p$-capacity on the Carnot groups, see Definition 14, and of the $p$-module of the family of curves connecting $S_{a}$ and $S_{b}$ in the spherical ring domain coincide, see [54]. We present here brief calculations of $M_{p}\left(\Gamma\left(\left(R_{a b} ; S_{a}, S_{b}\right)\right)\right)$ on a polarizable Carnot group, for the completeness.

We recall that if $c:[a, b] \rightarrow G$ is an absolutely continuous curve in a Carnot group $G$, which is not horizontal for some open subinterval $I \subset[a, b]$, then it is non-rectifiable. It was shown in [62] that even if $c$ is only continuous and rectifiable, then the tangent vector $\dot{c}(s)$ exists and it is horizontal for almost all $s \in[a, b]$. Thus, when computing the $p$-module of a family of curves, we can restrict ourselves to horizontal curves, because the $p$-module of a family 
of non-rectifiable curves vanishes [25]. Note also that any system of curves for $0<p<1$ has vanishing $p$-module [25].

Let $\phi$ be a solution to the Cauchy problem (20) satisfying the initial data $\phi(1, \xi)=\xi, \xi \in S_{1}$. The presence of the horizontal flow $\phi(\cdot, \xi):(0, \infty) \rightarrow G$, allows us to write the integral over $G$ in terms of spherical coordinates. Namely, the following proposition holds.

Proposition 8. 7] Let $G$ be a polarizable Carnot group. There exists a unique Radon measure $d v$ on $S_{1} \backslash \mathcal{Z}$, such that the integration formula

$$
\int_{G} f(g) d \mathbf{g}=\int_{S_{1} \backslash \mathcal{Z}} \int_{0}^{\infty} f(\phi(s, \xi)) s^{Q-1} d s d v(\xi)
$$

is valid for all $f \in L^{1}(G)$, where d $\mathbf{g}$ denotes the Haar measure on $G$.

Observe that the only information one needs to carry on the construction of spherical coordinates and forthcoming calculation of $M_{p}\left(\Gamma\left(\left(R_{a b} ; S_{a}, S_{b}\right)\right)\right)$ on a polarizable Carnot group is the existence of homogeneous norm $N_{G}$.

Theorem 5. Let $G$ be a polarazible Carnot group of Hausdorff dimension $Q$ with a homogeneous norm $N_{G}$ associated to Folland's solution to the Kohn subLaplacian. Let $\Gamma=\Gamma\left(R_{a b} ; S_{a}, S_{b}\right)$ be a family of horizontal locally rectifiable curves connecting the boundaries $S_{a}$ and $S_{b}$ in $R_{a b}$. Then, for $p>1$,

$$
M_{p}(\Gamma)=C_{S_{1}}(p) C_{a b}^{1-p}(p, Q),
$$

where

$$
C_{S_{1}}(p)=\int_{S_{1} \backslash \mathcal{Z}} \lambda^{p}(\xi) d v(\xi), \quad \text { and } \quad C_{a b}(p, Q):=\int_{a}^{b} s^{\frac{1-Q}{p-1}} d s .
$$

Proof. Let us use the integration in spherical coordinates (23) in order to calculate the module of $\Gamma$. For all admissible functions $\varrho$ we have

$$
1 \leqslant\left(\int_{\phi(\cdot, \xi)} \varrho\right)^{p}=\left(\int_{a}^{b} \varrho(\phi(s, \xi)) \lambda(\xi)^{-1} d s\right)^{p} \Longrightarrow \lambda(\xi) \leq \int_{\phi(\cdot, \xi)} \varrho,
$$

where $\lambda(\xi)^{-1}=\left\|\frac{d \phi(s, \xi)}{d s}\right\|_{0}=\frac{1}{\left\|\nabla_{0} N_{G}(\phi(s, \xi))\right\|_{0}}, \xi \in S_{1}$. Hölder's inequality implies

$$
\begin{aligned}
\lambda(\xi)^{p} \leqslant\left(\int_{a}^{b}\left(\varrho s^{\frac{Q-1}{p}}\right) s^{-\frac{Q-1}{p}} d s\right)^{p} \leqslant & {\left[\left(\int_{a}^{b} \varrho^{p} s^{Q-1} d s\right)^{\frac{1}{p}}\left(\int_{a}^{b} s^{\frac{1-Q}{p-1}} d s\right)^{\frac{p-1}{p}}\right]^{p} } \\
& =\left(\int_{a}^{b} \varrho^{p} s^{Q-1} d s\right)\left(\int_{a}^{b} s^{\frac{1-Q}{p-1}} d s\right)^{p-1} .
\end{aligned}
$$

Therefore

$$
\int_{a}^{b} \varrho^{p}(\phi(s, \xi)) s^{Q-1} d s \geqslant C_{a b}(p, Q)^{1-p} \lambda(\xi)^{p}
$$


EXTREMAL FUNCTIONS FOR MODULES...

and,

$$
\begin{aligned}
\int_{R_{a b}} \varrho^{p}(g) d \mathbf{g} & =\int_{S_{1} \backslash \mathcal{Z}} \int_{a}^{b} \varrho^{p}(\phi(s, \xi)) s^{Q-1} d s d v(\xi) \\
& \geq C_{a b}(p, Q)^{1-p} \int_{S_{1} \backslash \mathcal{Z}} \lambda(\xi)^{p} d v(\xi)=C_{a b}(p, Q)^{1-p} C_{S_{1}}(p)
\end{aligned}
$$

If we denote by $\Gamma_{0}$ the family of curves formed by the radial flow $\phi$, then $\Gamma_{0}$ is a subfamily of the family $\Gamma$. Taking infimum over admissible functions we obtain that

$$
M_{p}(\Gamma) \geq M_{p}\left(\Gamma_{0}\right) \geq C_{a b}(p, Q)^{1-p} C_{S_{1}}(p) .
$$

To find an estimation from above for $M_{p}(\Gamma)$ we present the extremal function on which this estimate is attained. It is given by

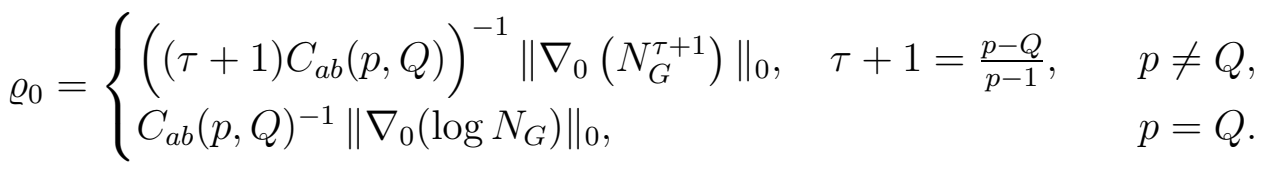

Considering $\varrho_{0}$ along the flow $\phi(s, \xi)$ of radial curves we obtain

$$
\varrho_{0}(\phi(s, \xi))=C_{a b}(p, Q)^{-1} s^{\tau} \lambda(\xi) .
$$

Using the integration in spherical coordinates (23) we calculate

$$
\begin{aligned}
\int_{R_{a b}} \varrho_{0}^{p} d \mathbf{g}= & \int_{S_{1} \backslash \mathcal{Z}} \int_{a}^{b} \varrho_{0}^{p}(\phi(s, \xi)) s^{Q-1} d s d v(\xi) \\
& =C_{a b}(p, Q)^{-p} \int_{S_{1} \backslash \mathcal{Z}} \int_{a}^{b} s^{\frac{p(1-Q)}{p-1}} \lambda(\xi)^{p} s^{Q-1} d s d v(\xi) \\
= & C_{a b}(p, Q)^{-p} \int_{S_{1} \backslash \mathcal{Z}} \lambda(\xi)^{p} d v(\xi) \int_{a}^{b} s^{\frac{1-Q}{p-1}} d s=C_{a b}(p, Q)^{1-p} C_{S_{1}}(p) .
\end{aligned}
$$

The function $\varrho_{0}$ is admissible for $\Gamma$ as it will be shown in the next Lemma 2 . Finally, we have

$$
M_{p}(\Gamma) \leqslant \int_{R_{a b}} \varrho_{0}^{p} d \mathbf{g}=C_{a b}(p, Q)^{1-p} C_{S_{1}}(p) .
$$

Lemma 2. For all $p>1$, the function $\varrho_{0}$, defined by (25) is admissible for the module $M_{p}(\Gamma)$ of a the family of curves connecting $S_{a}$ to $S_{b}$ in the spherical ring domain $R_{a b}$.

Proof. Let $\gamma:\left[0, l_{\gamma}\right] \rightarrow G$ be any curve in $\Gamma$ parametrized by arc-length: $\|\dot{\gamma}\|_{0}=$ 1, and such that $a=N(\gamma(0))$ and $b=N\left(\gamma\left(l_{\gamma}\right)\right)$. Then, by the Schwarz 
inequality we have $\left\langle\nabla_{0} N, \dot{\gamma}(s)\right\rangle_{0} \leqslant\left\|\nabla_{0} N\right\|_{0}$ for almost all $s \in\left[0, l_{\gamma}\right]$. It follows that for $p \neq Q$,

$$
\begin{aligned}
\int_{\gamma} \varrho_{0} & =\int_{0}^{l_{\gamma}} \varrho_{0}(\gamma(s)) d s \\
& =\left((\tau+1) C_{a b}(p, Q)\right)^{-1} \int_{0}^{l_{\gamma}}(\tau+1) N^{\tau}(\gamma(s))\left\|\nabla_{0} N(\gamma(s))\right\|_{0} d s \\
& \geq\left((\tau+1) C_{a b}(p, Q)\right)^{-1} \int_{0}^{l_{\gamma}}(\tau+1) N^{\tau}(\gamma(s))\left\langle\nabla_{0} N(\gamma(s)), \dot{\gamma}(s)\right\rangle d s \\
& =\left((\tau+1) C_{a b}(p, Q)\right)^{-1} \int_{0}^{l_{\gamma}} \frac{d}{d s} N^{\tau+1}(\gamma(s)) d s .
\end{aligned}
$$

Since $N^{\tau+1}(\gamma):\left[0, l_{\gamma}\right] \rightarrow R_{a b}$ is absolutely continuous, the Fundamental Theorem of Calculus results in

$$
\int_{\gamma} \varrho_{0} \geq\left((\tau+1) \int_{a}^{b} s^{\tau} d s\right)^{-1}\left(N^{\tau+1}\left(\gamma\left(l_{\gamma}\right)\right)-N^{\tau+1}(\gamma(0))\right)=1
$$

For $p=Q$, we obtain

$$
\begin{aligned}
\int_{\gamma} \varrho_{0} & =\int_{0}^{l_{\gamma}} \varrho_{0}(\gamma(s)) d s=\left(C_{a b}(p, Q)\right)^{-1} \int_{0}^{l_{\gamma}} N^{-1}(\gamma(s))\left\|\nabla_{0} N(\gamma(s))\right\|_{0} d s \\
& \geq\left(C_{a b}(p, Q)\right)^{-1} \int_{0}^{l_{\gamma}} N^{-1}(\gamma(s))\left\langle\nabla_{0} N(\gamma(s)), \dot{\gamma}(s)\right\rangle d s \\
& =\left(\int_{a}^{b} \frac{d s}{s}\right)^{-1} \int_{0}^{l_{\gamma}} \frac{d}{d s} \log N(\gamma(s)) d s=1 .
\end{aligned}
$$

Corollary 1. The family of radial curves $\Gamma_{0}$ satisfying (20) is the extremal family for the module $R_{a b}$ of the spherical ring domain $R_{a b}$ on polarizable Carnot groups. The function $\varrho_{0}$ given by (25) is the extremal function. Moreover, calculating the integral $C_{a b}(p, Q)^{1-p}$, we obtain

$$
M_{p}(\Gamma)= \begin{cases}C_{S_{1}}(p)\left(\frac{|p-Q|}{p-1}\right)^{p-1}\left|\left(b^{\frac{p-Q}{p-1}}-a^{\frac{p-Q}{p-1}}\right)\right|^{1-p}, & p \neq Q, \\ C_{S_{1}}(p)\left(\log \frac{b}{a}\right)^{1-Q}, & p=Q .\end{cases}
$$

As it was mentioned, the $H$-type groups are polarizable [7, Proposition 5.6], and the form of the homogeneous norm is given by (21). This allows us to calculate precisely the value of the constant $C_{S_{1}}(p)$ :

$$
C_{S_{1}}(p)=\int_{S_{1} \backslash \mathcal{Z}} \lambda(\xi)^{p} d v(\xi)=\frac{2 \pi^{k+l / 2} \Gamma\left(\frac{k+p}{4}\right)}{4^{l} \Gamma\left(\frac{k}{2}\right) \Gamma\left(\frac{k+2 l+p}{4}\right)},
$$

where $k=\operatorname{dim} V_{1}, l=\operatorname{dim} V_{2}$. The details can be found in [7]. 
4.3. The $p$-module of a family of separating sets in $R_{a b}$. We recall the definition of separating sets, given in Section 3.3. Unfortunately, some technical difficulties do not allow us to consider the separating sets in full generality in this section. Let $\Sigma=\Sigma\left(R_{a b} ; S_{a}, S_{b}\right)$ denote the class of all countably $H^{Q-1}$ rectifiable sets that separate $S_{a}$ from $S_{b}$ in $R_{a b} \subset G$. With every $\sigma \subset \Sigma$ we associate a complete measure $\mu$ in the following way: for every Hausdorff $H^{Q-1}$-measurable set $A \subset G$ define

$$
\mu(A)=\mathcal{H}_{H}^{Q-1}\left(A \cap \sigma \cap R_{a b}\right),
$$

where $Q$ is the Hausdorff dimension of the group $G$. Let $E$ denote the family of such measures associated with $\Sigma$. Let us describe this measures on spheres $S_{s}$ in details. The integration formula (23) implies that the volume element $d \mathbf{g}$ along the flow defined by $\phi$ can be written as

$$
d \mathbf{g}=s^{Q-1} d s d v(\xi)=s^{Q-1}\left\|\frac{\partial \phi}{\partial s}\right\|_{0}^{-1} d v(\xi)\left\|\frac{\partial \phi}{\partial s}\right\|_{0} d s=s^{Q-1} \lambda(\xi) d v(\xi) \lambda(\xi)^{-1} d s .
$$

Therefore, the measure $d S_{1}(\xi)=\lambda(\xi) d v(\xi), \xi \in S_{1}$ is absolutely continuous with respect to the Radon measure $d v(\xi)$ and represents an $H^{Q-1}$ dimensional surface measure on the unit sphere $S_{1}$. The element of the surface area on the sphere $S_{s}$ of radius $s$ is given by $d S_{s}=s^{Q-1} \lambda(\xi) d v(\xi)$. The part $d \phi=\lambda(\xi)^{-1} d s$ defines the element of length of the curve $\phi(\cdot, \xi)$. In the case $G=\mathbb{R}^{k}$, we obtain that $\lambda(\xi) \equiv 1$, and $d S_{1}=d v$ is the usual surface element on the unit sphere.

Theorem 6. Let $G$ be a polarazible Carnot group of Hausdorff dimension $Q$ with the homogeneous norm $N_{G}$ associated to Folland's solution to the Kohn sub-Laplacian. Let $E$ be the family of measures associated with sets separating $S_{a}$ and $S_{b}$ in $R_{a b}$. Then for $q>1$ we obtain

$$
M_{q}(E)=K_{a b}(q, Q) K_{S_{1}}^{1-q}(q),
$$

where

$$
K_{S_{1}}(q)=\int_{S_{1} \backslash \mathcal{Z}} \lambda^{\frac{q}{q-1}}(\xi) d v(\xi), \quad K_{a b}(q, Q)=\int_{a}^{b} s^{(1-q)(Q-1)} d s .
$$

Proof. Let $\rho$ be an admissible function for the family $E$. Then, for any sphere $S_{s}, a<s<b$ we have

$$
\begin{aligned}
1 & \leq\left(\int_{S_{s} \backslash \mathcal{Z}} \rho(\phi(s, \xi)) d S_{s}\right)^{q}=\left(\int_{S_{1} \backslash \mathcal{Z}} s^{Q-1} \rho(\phi(s, \xi)) \lambda(\xi) d v(\xi)\right)^{q} \\
& \leq s^{q(Q-1)}\left(\int_{S_{1} \backslash \mathcal{Z}} \rho^{q}(\phi(s, \xi)) d v(\xi)\right)\left(\int_{S_{1} \backslash \mathcal{Z}} \lambda^{\frac{q}{q-1}}(\xi) d v(\xi)\right)^{q-1} .
\end{aligned}
$$

Thus,

$$
\int_{S_{1} \backslash \mathcal{Z}} \rho^{q}(\phi(s, \xi)) d v(\xi) \geq s^{-q(Q-1)}\left(\int_{S_{1} \backslash \mathcal{Z}} \lambda^{\frac{q}{q-1}}(\xi) d v(\xi)\right)^{1-q}
$$


Then, we arrive at the inequality

$$
\int_{R_{a b}} \rho^{q} d \mathbf{g}=\int_{a}^{b} s^{Q-1} d s \int_{S_{1} \backslash \mathcal{Z}} \rho^{q} d v \geq \int_{a}^{b} s^{(1-q)(Q-1)}\left(\int_{S_{1} \backslash \mathcal{Z}} \lambda^{\frac{q}{q-1}}(\xi) d v(\xi)\right)^{1-q} .
$$

Making use of notations (26), we come to a lower bound for the module $M_{q}(E)$ of the family of separating sets

$$
M_{q}(E) \geq M_{q}\left(E_{0}\right) \geq K_{a b}(q, Q) K_{S_{1}}(q)^{1-q},
$$

where $E_{0}$ is the family of measures associated with the spheres $S_{s}=\{g \in G \mid$ $\left.N_{G}(g)=s\right\}$ for $a<s<b$ which separate the boundaries of the spherical ring domain $R_{a b}$.

Now we turn to the estimation of $M_{q}(E)$ from above. The extremal function in this case is given by the following expression

$$
\rho_{0}(g)= \begin{cases}(\tau+1)^{\frac{1}{1-q}} K_{S_{1}}^{-1}(q)\left\|\nabla_{0}\left(N_{G}^{\tau+1}(g)\right)\right\|_{0}^{\frac{1}{q-1}}, \tau=(q-1)(1-Q), & q \neq \frac{Q}{Q-1}, \\ K_{S_{1}}^{-1}(q)\left\|\nabla_{0}\left(\log N_{G}(g)\right)\right\|_{0}^{\frac{1}{q-1}}, & q=\frac{Q}{Q-1} .\end{cases}
$$

Restricting the value of $\rho_{0}$ to the sphere $N_{G}(g)=s$ we conclude that

$$
\rho_{0}(\varphi(s, \xi))=\rho_{0}(g)=K_{S_{1}}^{-1}(q) s^{1-Q} \lambda^{\frac{1}{q-1}}(\xi) .
$$

Thus,

$$
\int_{R_{a b}} \rho_{0}^{q} d \mathbf{g}=K_{S_{1}}^{-q}(q) \int_{a}^{b} s^{Q-1+q(1-Q)} d s \int_{S_{1} \backslash \mathcal{Z}} \lambda^{\frac{q}{q-1}} d v(\xi)=K_{a b}(q, Q) K_{S_{1}}^{1-q}(q) .
$$

The function $\rho_{0}$ is admissible for the family of separating sets as it will be proved in Subsection 4.4. Finally, taking the infimum over the admissible functions, we obtain

$$
M_{q}(E) \leq \int_{R_{a b}} \rho_{0}^{q} d \mathbf{g}=K_{a b}(q, Q) K_{S_{1}}^{1-q}(q)
$$

This finishes the proof.

Corollary 2. The family of measures $E_{0}$ associated with the spheres $\Sigma_{0}=$ $\left\{S_{s}, a<s<b\right\}$ is the extremal family for the module $M_{q}(E)$ of sets $\Sigma$ separating the spheres $S_{a}$ and $S_{b}$ in the spherical ring domain $R_{a b}$ on polarizable Carnot groups. The function $\rho_{0}$ given by (27) is extremal. In particular, $\int_{S_{s}} \rho_{0} d S_{s}=1$ for any sphere $S_{s}, a<s<b$.

Let us observe the following relations that reveal Theorems 5 and 6 .

Corollary 3. For $\frac{1}{p}+\frac{1}{q}=1$, Theorems 5 and 6 imply

1. $K_{a b}(q, Q)=C_{a b}(p, Q)$,

2. $K_{S_{1}}(q)=C_{S_{1}}(p)$, 
3. $M_{p}^{\frac{1}{p}}(\Gamma) M_{q}^{\frac{1}{q}}(\Sigma)=1$,

4. $\rho_{0}=C_{a b}^{p-1}(p, Q) C_{S_{1}}^{-1}(p) \varrho_{0}^{p-1}, p \neq Q$

4.3.1. Relations between $M_{p}(\Gamma), M_{q}(E)$, and the capacity $\operatorname{cap}_{p}\left(\mathbb{R}_{a b}\right)$. Before we proceed to show that the function $\rho_{0}$ is admissible for the family $E$, we review the relations between $M_{p}(\Gamma), M_{q}(E)$, and the capacity cap $\left.\cos _{p b}\right)$.

Definition 14. Let $\Omega$ be a domain in $G$, and let $D_{0}, D_{1}$ be two disjoint compacts in the closure $\bar{\Omega}$ of $\Omega$. A function $u \in W^{1, p}(\Omega)$, such that $\left.u\right|_{D_{0}}=0$ and $\left.u\right|_{D_{1}}=1$, is called admissible for the condenser $\left(\Omega ; D_{0}, D_{1}\right)$. The value

$$
\operatorname{cap}_{p}\left(\Omega ; D_{0}, D_{1}\right)=\inf \int_{\Omega}\left\|\nabla_{0} u\right\|_{0}^{p} d x,
$$

is called a p-capacity of the condenser $\left(\Omega ; D_{0}, D_{1}\right)$, where the infimum is taken over all admissible functions $u$.

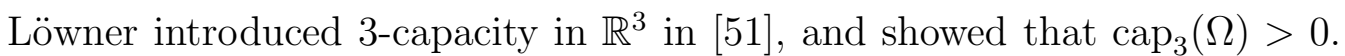
Gehring [29] proved that the Löwner 3-capacity (or conformal capacity) for a ring domain in $\mathbb{R}^{3}$, coincides with the module $M_{3}(\Gamma)$ of a family of curves, which was calculated by Väisälä earlier in [85], and that it is also equal to the module $M_{3 / 2}(E)^{-2}$ of the family of surface measures on compact piecewise smooth surfaces $\Sigma$ separating $D_{0}$ and $D_{1}$ in a bounded domain $\Omega \subset \mathbb{R}^{3}$. The latter notion was used by Šabat [76] in his study of quasiconformal maps in $\mathbb{R}^{3}$. The restriction to smooth surfaces was relaxed in [48] provided that admissible functions behave sufficiently nice. Later in 1966-68, Zimmer showed that the module $M_{n}(\Gamma)$ of a family of curves connecting $D_{0}$ and $D_{1}$ in a bounded domain $\Omega \subset \mathbb{R}^{n}$ is in the following relation with the module $M_{\frac{n}{n-1}}(E)$ of the family of measures associated with the sets separating $D_{0}$ and $D_{1}$ [88]:

$$
\left(M_{n}(\Gamma)\right)^{\frac{1}{n}}\left(M_{\frac{n}{n-1}}(E)\right)^{\frac{n-1}{n}}=1 .
$$

In order to relax the conditions on admissible functions, the method of symmetrisaition in [27] and surface-theoretical approximation theorems permit to consider general separating sets, see [22]. Shlyk showed in [74], that for a rather general condenser $\left(\Omega ; D_{0}, D_{1}\right)$ in $\mathbb{R}^{n}$, the equality (28) can be extended as follows

$$
\left(M_{p}(\Gamma)\right)^{\frac{1}{p}}\left(M_{q}(E)\right)^{\frac{1}{q}}=1, \quad \frac{1}{p}+\frac{1}{q}=1 .
$$

For further interesting generalizations for modules in $\mathbb{R}^{n}$ see [6]. Some extensions to the Carnot groups can be found in [55, 56, 57], and for arbitrary metric measure spaces for instance in [73].

Zimmer proved [89, 90], that the capacity $\operatorname{cap}_{p}\left(\Omega ; D_{0}, D_{1}\right)$ coincides with the module $M_{p}\left(\Gamma\left(\Omega ; D_{0}, D_{1}\right)\right)$ of a family of curves connecting $D_{0}$ and $D_{1}$ in 
$\Omega$, where the domain $\Omega \subset \mathbb{R}^{n}$ is assumed to be bounded. Hesse [39] extended his result to unbounded domains. In particular, he showed that the set of admissible functions for the $p$-module of a family of curves can be restricted from non-negative Borel measurable functions in $\mathbb{R}^{n}$ to lower semicontinuous $L_{p}$-functions in $\mathbb{R}^{n}$, which are continuous in $\Omega$, provided that $D_{0} \cup D_{1} \subset \Omega$. Shlyk [75] generalized the result of Hesse from a connected open set (domain) $\Omega$ to an arbitrary open set in $\overline{\mathbb{R}}^{n}$.

In general, the relation between the admissible function $u$ for the $p$-capacity of a condenser $\left(\Omega ; D_{0}, D_{1}\right)$, and the admissible function $\rho$ for the $p$-module of a family of curves connecting $D_{0}$ and $D_{1}$ is as follows. Let $\rho$ be an admissible function for the family of curves connecting $D_{0}$ and $D_{1}$. Then the function $u(x)=\min \left\{1\right.$, inf $\left.\int_{\beta_{x}} \rho\right\}$ is admissible for the $p$-capacity of the condenser $\left(\Omega ; D_{0}, D_{1}\right)$, where the infimum is taken over all locally rectifiable curves $\beta_{x}$ in $\Omega$ connecting $D_{0}$ and the point $x \in \Omega$. Moreover,

$$
|\nabla u| \leq \rho \quad \text { almost everywhere in } \Omega
$$

This immediately implies the inequality

$$
\operatorname{cap}_{p}\left(\Omega ; D_{0}, D_{1}\right) \leq \int_{\mathbb{R}^{n}}|\nabla u|^{p} d x \leq \int_{\mathbb{R}^{n}} \rho^{p} d x \leq M_{p}(\Gamma)
$$

by taking infimum over all admissible functions $\rho$ for the $p$-module. On the other hand, if $u$ is an admissible $W^{1, p}$-function for the $p$-capacity of $\left(\Omega ; D_{0}, D_{1}\right)$, then

$$
\rho(x)= \begin{cases}|\nabla u(x)|, & x \in \Omega, \\ 0, & x \in \mathbb{R}^{n} \backslash \Omega\end{cases}
$$

is an admissible function for the module $M_{p}(\Gamma)$ of the family of curves connecting $D_{0}$ and $D_{1}$, that implies the inequality

$$
M_{p}(\Gamma) \leq \int_{\mathbb{R}^{n}} \rho^{p} d x=\int_{\mathbb{R}^{n}}|\nabla u|^{p} d x \leq \operatorname{cap}_{p}\left(\Omega ; D_{0}, D_{1}\right)
$$

upon taking infimum over all admissible functions $u$ for the $p$-capacity.

Let us also mention a relation between the extremal functions $\varrho_{0}, \rho_{0}$ for the modules $M_{p}(\Gamma)$ and $M_{q}(E)$, and the extremal function $u$ for the $p$-capacity of the condenser $\left(R_{a b} ; S_{a}, S_{b}\right)$. It is well known that the variational equation for the problem of finding the $p$-capacity on a polarizable Carnot group $G$ (and particularly in $\mathbb{R}^{n}$ ) is the $p$-sub-Laplacian equation, and the extremal function $u$ for the $p$-capacity is a solution to the $p$-sub-Laplace equation in $G$ with prescribed boundary values on $D_{0}$ and $D_{1}$. It was shown [7], that the function

$$
\tilde{u}(g)= \begin{cases}c_{p} N_{G}^{\tau+1}, \quad \tau+1=\frac{p-Q}{p-1}, & \text { for } p \neq Q \\ c_{Q} \log N_{G}, & \text { for } p=Q\end{cases}
$$


is a fundamental solution to the $p$-sub-Laplacian equation on $G$ for some appropriate choice of constants, see [17] for an analogous result on $H$-type groups. One can easily check that

$$
u(g)=\frac{N_{G}^{\tau+1}(g)-a^{\tau+1}}{b^{\tau+1}-a^{\tau+1}}, \quad g \in R_{a b}
$$

is extremal for the $p$-capacity of $\left(R_{a b} ; S_{a}, S_{b}\right)$.

4.4. Admissibility of $\rho_{0}$ for $M_{q}(E)$. In this section we will show that the function $\rho_{0}$ defined in (27) is admissible for a system $E$ of Hausdorff measures $H^{Q-1}$ associated with a family $\Sigma$ of countably $H^{Q-1}$-rectifiable sets separating $S_{a}$ and $S_{b}$ in $R_{a b}$. The core idea of the proof is to show that if $u$ is an extremal function for the $p$-capacity of $\left(R_{a b} ; S_{a}, S_{b}\right)$, then $\left\|\nabla_{0} u\right\|_{0}^{p-1}$ is an admissible function for $M_{q}(E)$ with $\frac{1}{p}+\frac{1}{q}=1$. This method goes back to Gehring [29] who proved a similar result for $\mathbb{R}^{3}$, which was extended for the $n$-capacities and $n$-modules by Ziemer [88] in $\mathbb{R}^{n}$. Later, Shlyk 74] generalized the proof to $\mathbb{R}^{n}$ for arbitrary values of $p \neq n$. The same result was implicitly presented in [6] for $\mathbb{R}^{n}$, and in [54] for arbitrary Carnot groups. Here we want to follow the ideas of Gehring [29]. We emphasize that in $\mathbb{R}^{n}$ the result was obtained for arbitrary system of separating sets. The lack of approximations theorems, such as, for instance [88, Theorem 2.4.2], does not allow us to extend the proof of Theorem 7 to arbitrary separating sets on polarizable groups. Our main goal is to show the following theorem.

Theorem 7. Let $\Sigma$ be a family of countably $H^{Q-1}$-rectifiable sets separating $S_{a}$ and $S_{b}$ in $R_{a b}, \sigma \in \Sigma$, and let $u$ be an extremal function for the p-capacity of $\left(R_{a b}, S_{a}, S_{b}\right)$. Let $E$ be a family of $(Q-1)$-Hausdorff measures $H^{Q-1}$ associated with $\Sigma$. Then the integral $\int_{\sigma}\left\|\nabla_{0} u(g)\right\|_{0}^{p-1} d H^{Q-1}(g)$ exists for $M_{q}(E)$-almost all measures from $E$ and

$$
\int_{\sigma}\left\|\nabla_{0} u(g)\right\|_{0}^{p-1} d H^{Q-1}(g) \geq \operatorname{cap}_{p}\left(R_{a b} ; S_{a}, S_{b}\right), \quad \frac{1}{p}+\frac{1}{q}=1 .
$$

The proof is forestalled by two lemmas. Before we formulate the statement of the first lemma let us describe some constructions which we will use. Let $\sigma \in \Sigma$, and let $\beta>0$ be such that $\beta<\operatorname{dist}\left(\sigma, \partial R_{a b}\right)$. We denote by $\sigma(\beta)=$ $\left\{g \in R_{a b} \mid \operatorname{dist}(\mathfrak{g}, \sigma)<\beta\right\}$ and by $d(g)=\operatorname{dist}(g, \sigma)$. Here the distance is understood as $\operatorname{dist}(g, \eta)=N_{G}\left(\eta^{-1} g\right), g, \eta \in G$. By construction, the norm $N_{G}=u_{2}^{\frac{1}{2-Q}}$ is smooth away from the identity of $G$ due to the smoothness of the solution $u_{2}$. This guarantees that the function $d$ is at least Lipschitz in $G$. 
Lemma 3. Let $u$ be an extremal function for the p-capacity of the condenser $\left(R_{a b} ; S_{a}, S_{b}\right)$, and let $\sigma \in \Sigma$. Then

$$
\int_{\sigma(\beta)}\left\|\nabla_{0} u(g)\right\|_{0}^{p-1}\left\|\nabla_{0} d(g)\right\|_{0} d \mathbf{g} \geq 2 \beta \operatorname{cap}_{p}\left(R_{a b} ; S_{a}, S_{b}\right) .
$$

Proof. Denote by $\bar{R}_{a b}$ the closure of $R_{a b}$, and by $A^{c}$ the complement to $A$ in $G$. Let $F_{0}$ be a component of $\sigma^{c} \cap \bar{R}_{a b}$ containing $S_{a}$, and let $F_{1}$ be a component of $\sigma^{c} \cap \bar{R}_{a b}$ containing $S_{b}$. Let

$$
E_{k}=\left\{g \in \bar{R}_{a b} \mid 0<\operatorname{dist}\left(g, F_{k}^{c}\right)<\beta\right\}, \quad k=0,1 .
$$

Then $E_{k} \subset F_{k}, E_{0} \cup E_{1} \subset \sigma(\beta)$, and it is sufficient to show that

$$
\int_{E_{k}}\left\|\nabla_{0} u(g)\right\|_{0}^{p-1}\left\|\nabla_{0} d(g)\right\|_{0} d \mathbf{g} \geq \beta \operatorname{cap}_{p}\left(R_{a b} ; S_{a}, S_{b}\right), \quad k=0,1 .
$$

We focus ourselves only on the case $k=0$. The case $k=1$ is treated analogously. Define

$$
v(g)=\min \left\{\beta, \operatorname{dist}\left(g, F_{0}^{c}\right)\right\}= \begin{cases}0, & \text { if } g \in F_{0}^{c}=F_{1} \cup \sigma \\ \inf _{\eta \in F_{0}^{c}} \operatorname{dist}(g, \eta) & \text { if } g \in E_{0} \\ \beta & \text { if } g \in F_{0} \backslash E_{0} .\end{cases}
$$

The function $v$ is Lipschitz, from the class $L^{p}\left(R_{a b}\right)$, and

$$
\left\|\nabla_{0} v(g)\right\|_{0}=\left\{\begin{array}{lc}
\left\|\nabla_{0} d(g)\right\|_{0}>0 & \text { almost everywhere in } E_{0}, \\
0 & \text { in } R_{a b} \backslash E_{0} .
\end{array}\right.
$$

Thus, the function $w=v-\beta u$ is almost everywhere differentiable and belongs to the class $L^{p}\left(R_{a b}\right)$. We use $w$ as a test function on $R_{a b}$ and obtain

$0=\int_{R_{a b}}\left\|\nabla_{0} u(g)\right\|_{0}^{p-2}\left\langle\nabla_{0} u, \nabla_{0} w\right\rangle_{0} d \mathbf{g}=\int_{R_{a b}}\left\|\nabla_{0} u(g)\right\|_{0}^{p-2}\left\langle\nabla_{0} u, \nabla_{0} v-\beta \nabla_{0} u\right\rangle_{0} d \mathbf{g}$.

This, together with the Cauchy-Schwartz inequality, implies

$$
\begin{aligned}
\int_{E_{0}}\left\|\nabla_{0} u(g)\right\|_{0}^{p-1}\left\|\nabla_{0} d(g)\right\|_{0} d \mathbf{g} & =\int_{R_{a b}}\left\|\nabla_{0} u(g)\right\|_{0}^{p-1}\left\|\nabla_{0} v(g)\right\|_{0} d \mathbf{g} \\
& \geq \int_{R_{a b}}\left\|\nabla_{0} u(g)\right\|_{0}^{p-2}\left\langle\nabla_{0} u(g), \nabla_{0} v(g)\right\rangle_{0} d \mathbf{g} \\
& =\beta \int_{R_{a b}}\left\|\nabla_{0} u(g)\right\|_{0}^{p} d \mathbf{g}=\beta \operatorname{cap}_{p}\left(R_{a b} ; S_{a}, S_{b}\right) .
\end{aligned}
$$

If we were pass to the limit in

$$
\frac{1}{2 \beta} \int_{\sigma(\beta)}\left\|\nabla_{0} u(g)\right\|_{0}^{p-1}\left\|\nabla_{0} d(g)\right\| d \mathbf{g} \geq \operatorname{cap}_{p}\left(R_{a b} ; S_{a}, S_{b}\right)
$$


as $\beta \rightarrow 0$, we could finish the proof of Theorem 7 at once. In order to show that the limit exists, we consider the sequences of continuous functions $f_{r}(g)$ converging to $\left\|\nabla_{0} u(g)\right\|_{0}^{p-1}$ g-almost everywhere as $r \rightarrow 0$ and such that the limit

$$
\frac{1}{2 \beta} \int_{\sigma(\beta)} f_{r}(g)\left\|\nabla_{0} d(g)\right\| d \mathbf{g} \rightarrow \int_{\sigma} f_{r}(g) d H^{Q-1} \quad \text { as } \quad \beta \rightarrow 0
$$

exists. We define the integral mean of $\left\|\nabla_{0} u(g)\right\|_{0}^{p-1}$ in the ball by

$$
f_{r}(g)=\frac{1}{\mathbf{g}(B(g, r))} \int_{B(g, r)}\left\|\nabla_{0} u(\eta)\right\|_{0}^{p-1} d \mathbf{g}(\eta) .
$$

We also recall the co-area formula for Carnot groups. Let $U$ be a domain in $G$. Let $f \in L^{1}(U)$ be a non-negative function, and let $v$ be a real valued Lipschitz function in $U$, see [36, 44, 52]. Then

$$
\int_{U} f(g)\left\|\nabla_{0} v(g)\right\|_{0} d \mathbf{g}(g)=\int_{-\infty}^{+\infty} \int_{v^{-1}(s)} f(\eta) d H^{Q-1}(\eta) d s .
$$

Lemma 4. The integral mean (31) satisfies the inequality

$$
\int_{\sigma} f_{r}(g) d H^{Q-1} \geq \operatorname{cap}_{p}\left(R_{a b} ; S_{a}, S_{b}\right)
$$

whenever $r<\operatorname{dist}\left(\sigma, \partial R_{a b}\right)$.

Proof. If $H^{Q-1}(\sigma)=\infty$, then there is nothing to prove. Assume that $H^{Q-1}(\sigma)<$ $\infty$. Let $\beta, r$ be positive numbers such that $\beta+r<\operatorname{dist}\left(\sigma, \partial R_{a b}\right)$. Let $L_{\eta}(\sigma)=\eta \sigma$ be a left translation of the set $\sigma$ by an element $\eta \in G$. Then, changing variables and using Fubini's theorem we come to

$$
\begin{aligned}
& \int_{\sigma(\beta)} f_{r}(g)\left\|\nabla_{0} d(g)\right\|_{0} d \mathbf{g}(g) \\
= & \frac{1}{\mathbf{g}(B(g, r))} \int_{B(0, r)} d \mathbf{g}(\eta) \int_{\sigma(\beta)}\left\|\nabla_{0} u(\eta g)\right\|_{0}^{p-1}\left\|\nabla_{0} d(g)\right\|_{0} d \mathbf{g}(g) .
\end{aligned}
$$

Observe that $d(g)=\operatorname{dist}(g, \sigma)=\operatorname{dist}(\eta g, \eta \sigma)=\operatorname{dist}(\psi, \eta \sigma)$, with $\psi=\eta g$. Then making change of variables $\psi=\eta g$, we write the last integral in the form

$$
\begin{aligned}
& \frac{1}{\mathbf{g}(B(g, r))} \int_{B(0, r)} d \mathbf{g}(\eta) \int_{\eta \sigma(\beta)}\left\|\nabla_{0} u(\psi)\right\|_{0}^{p-1}\left\|\nabla_{0} \operatorname{dist}(\psi, \eta \sigma)\right\|_{0} d \mathbf{g}(\psi) \\
= & \frac{1}{\mathbf{g}(B(g, r))} \int_{B(0, r)} d \mathbf{g}(\eta) \int_{(\eta \sigma)(\beta)}\left\|\nabla_{0} u(\psi)\right\|_{0}^{p-1}\left\|\nabla_{0} d(\psi)\right\|_{0} d \mathbf{g}(\psi) \\
\geq & 2 \beta \operatorname{cap}_{p}\left(R_{a b} ; S_{a}, S_{b}\right),
\end{aligned}
$$


where the last inequality follows from Lemma 3. Moreover, applying the coarea formula (32), we obtain

$$
\int_{\sigma(\beta)} f_{r}(g)\left\|\nabla_{0} d(g)\right\|_{0} d \mathbf{g}(g)=\int_{0}^{\beta} \int_{d^{-1}(s)} f_{r}(\zeta) d H^{Q-1}(\zeta) d s .
$$

Let $F(s)$ denote the interior integral in the right-hand side,

$$
F(s)=\int_{d^{-1}(s)} f_{r}(\zeta) d H^{Q-1}(\zeta)
$$

The function $f_{r}$ is continuous on $G$, and the set $\sigma$ is countably rectifiable. Then

$$
F(s)=\int_{d^{-1}(s)} f_{r}(\zeta) d H^{Q-1}(\zeta) \rightarrow 2 F(0)=2 \int_{\sigma} f_{r}(\zeta) d H^{Q-1}(\zeta), \quad \text { as } \quad s \rightarrow 0 .
$$

Thus gathering the above results we arrive at

$$
\begin{aligned}
\operatorname{cap}_{p}\left(R_{a b} ; S_{a}, S_{b}\right) & \leq \lim _{\beta \rightarrow 0} 2 \frac{1}{2 \beta} \int_{\sigma(\beta)} f_{r}(g)\left\|\nabla_{0} d(g)\right\|_{0} d \mathbf{g}(g) \\
& =\lim _{\beta \rightarrow 0} \frac{1}{\beta} \int_{0}^{\beta} F(s) d s=\int_{\sigma} f_{r}(\zeta) H^{Q-1}(\zeta) .
\end{aligned}
$$

Proof of Theorem 17. Let $\sigma \in \Sigma$. We can assume that for any $r<r_{0}<$ $\operatorname{dist}\left(\partial F_{0}, \partial R_{a b}\right)$, the support of $f_{r}$ belongs to $R_{a b}$. Then,

$$
f_{r} \rightarrow\left\|\nabla_{0} u\right\|_{0}^{p-1} \mathbf{g}-\text { almost everywhere as } r \rightarrow 0,
$$

and $\int_{R_{a b}} f_{r}^{q} d \mathbf{g} \leq \int_{R_{a b}}\left\|\nabla_{0} u\right\|_{0}^{p} d \mathbf{g}<\infty$. The Lebesgue dominated convergence theorem implies that $f_{r}$ converges to $\left\|\nabla_{0} u\right\|_{0}^{p-1}$ in $L^{q}\left(R_{a b}\right)$ as $r \rightarrow 0$. Therefore, there is a subsequence (that we will denote by the same symbol) $f_{r}$, such that

$$
\int_{\sigma}\left|f_{r}-\left\|\nabla_{0} u\right\|_{0}^{p-1}\right| d H^{Q-1} \rightarrow 0 \quad \text { as } \quad r \rightarrow 0,
$$

for $M_{q}(E)$-almost all measures $\mu \in E$. Thus, the integral

$$
\int_{\sigma}\left\|\nabla_{0} u\right\|_{0}^{p-1} d H^{Q-1}
$$

exists and moreover inequality (30) holds.

Corollary 4. The function $\rho_{0}$ defined in (27) is admissible for the module $M_{q}(E)$ of the family of measures associated with sets separating $S_{a}$ and $S_{b}$ in $R_{a b}$. 
Proof. As it was mentioned, the function

$$
u(g)=\frac{N_{G}^{\tau+1}(g)-a^{\tau+1}}{b^{\tau+1}-a^{\tau+1}}, \quad g \in G,
$$

is extremal for $\operatorname{cap}_{p}\left(R_{a b} ; S_{a}, S_{b}\right)$ and $\varrho_{0}=\left\|\nabla_{0} u\right\|_{0}$ by (25). Therefore,

$$
\operatorname{cap}_{p}\left(R_{a b} ; S_{a}, S_{b}\right)=\int_{R_{a b}}\left\|\nabla_{0} u\right\|_{0}^{p} d \mathbf{g}=\int_{R_{a b}} \varrho_{0}^{p} d \mathbf{g}=M_{p}(\Gamma),
$$

where $\Gamma$ is the family of all locally rectifiable curves connecting $S_{a}$ and $S_{b}$. Moreover, $\rho_{0}=C_{a b}^{p-1}(p, Q) C_{S_{1}}^{-1}(p)\left\|\nabla_{0} u\right\|_{0}^{p-1}$ as shows (27). Thus, for any $\sigma \in \Sigma$ we obtain

$$
\begin{aligned}
\int_{\sigma} \rho_{0} d H^{Q-1} & =C_{a b}^{p-1}(p, Q) C_{S_{1}}^{-1}(p) \int_{\sigma}\left\|\nabla_{0} u\right\|_{0}^{p-1} d H^{Q-1} \\
& \geq C_{a b}^{p-1}(p, Q) C_{S_{1}}^{-1}(p) \operatorname{cap}_{p}\left(R_{a b}, S_{a}, S_{b}\right) \\
& \geq C_{a b}^{p-1}(p, Q) C_{S_{1}}^{-1}(p) M_{p}(\Gamma)=1
\end{aligned}
$$

4.5. Twisting map of the spherical ring in the Heisenberg group. Let us consider the spherical ring $R_{1 b}=\left\{\left(x_{1}, x_{2}, t\right): 1 \leq N_{\mathbb{H}}\left(x_{1}, x_{2}, t\right) \leq b\right\}$ in the Heisenberg group $\mathbb{H}=\mathbb{H}^{1}$ with respect to the homogeneous norm $N_{\mathbb{H}}$. The coordinates in $\mathbb{H}$ are $\left(x_{1}, x_{2}, t\right)$, or in the polar form $(\theta, \alpha, r),\left(x_{1}, x_{2}, t\right)=$ $G(\theta, \alpha, r)$, where $G:(\theta, \alpha, r) \rightarrow\left(x_{1}, x_{2}, t\right)$ is given by

$$
x_{1}=r \sqrt{\cos \alpha} \cos \theta, \quad x_{2}=r \sqrt{\cos \alpha} \sin \theta, \quad t=r^{2} \sin \alpha,
$$

$\theta \in[0,2 \pi), \alpha \in(-\pi / 2, \pi / 2), r \in[1, b]$. The horizontal vector fields in the polar form are

$$
\begin{aligned}
& X_{1}=\frac{\partial}{\partial x_{1}}+2 x_{2} \frac{\partial}{\partial t}=\sqrt{\cos \alpha}\left(\cos (\theta-\alpha) \frac{\partial}{\partial r}+\frac{2}{r} \sin (\theta-\alpha) \frac{\partial}{\partial \alpha}-\frac{\sin \theta}{r \cos \alpha} \frac{\partial}{\partial \theta}\right), \\
& X_{2}=\frac{\partial}{\partial x_{2}}-2 x_{1} \frac{\partial}{\partial t}=\sqrt{\cos \alpha}\left(\sin (\theta-\alpha) \frac{\partial}{\partial r}-\frac{2}{r} \cos (\theta-\alpha) \frac{\partial}{\partial \alpha}+\frac{\cos \theta}{r \cos \alpha} \frac{\partial}{\partial \theta}\right) .
\end{aligned}
$$

The horizontal norm of the horizontal gradient of a smooth function $f(\theta, \alpha, r)$ is calculated as $\left\|\nabla_{0} f\right\|_{0}=\left(X_{1}^{2}(f)+X_{2}^{2}(f)\right)^{1 / 2}$. The $H^{3}$-Hausdorff measure element on the sphere $S_{r} \backslash \mathcal{Z}$ is $d \omega_{r}=r^{3} \sqrt{\cos \alpha} d \alpha d \theta$. In particular, the area of the unit sphere $S_{1}$ is calculated as

$$
\operatorname{Area}\left(S_{1}\right)=4 \sqrt{2 \pi} \Gamma^{2}\left(\frac{3}{4}\right)
$$

where Euler's $\Gamma$-function is $\Gamma(x)=\int_{0}^{\infty} t^{x-1} e^{-t} d t$. The radial flow $\phi(r, \theta, \alpha)$ on $\mathbb{H}$ orthogonal to the sphere $S_{1} \backslash \mathcal{Z}$ is a solution to the initial-value problem (20) 
in the particular case of the homogeneous Heisenberg norm $N_{\mathbb{H}}$, given by

$$
\begin{aligned}
x_{1}(r) & =r \sqrt{\cos \alpha} \cos (\theta-\tan \alpha \log r), \\
x_{2}(r) & =r \sqrt{\cos \alpha} \sin (\theta-\tan \alpha \log r), \\
t(r) & =r^{2} \sin \alpha,
\end{aligned}
$$

where $1 \leq r \leq b$, and $\theta \in[0,2 \pi), \alpha \in(-\pi / 2, \pi / 2)$ are fixed. The horizontal norm of $\dot{\phi}(r, \theta, \alpha)=\frac{\partial}{\partial r} \phi(r, \theta, \alpha)$ is $\|\dot{\phi}(r, \theta, \alpha)\|_{0}=\cos ^{-1 / 2} \alpha$.

An analogue to Theorem 3 can be formulated for the spherical ring domain in $\mathbb{H}$ as follows. Let $\Gamma_{0}$ denote the family of curves $\phi_{\theta \alpha}(\cdot):[1, b] \rightarrow \mathbb{H}$ given by radial flow $\phi(r, \theta, \alpha)$ for every fixed $\alpha \in(-\pi / 2, \pi / 2), \theta \in[0,2 \pi)$. In order to preserve the horizontal nature of the families of curves we require from a smooth map $f: \mathbb{H} \rightarrow \mathbb{H}$ to be the contact map, that is a map whose differential preserves the horizontal planes span $\left\{X_{1}(g), X_{2}(g)\right\}$ for all $g \in \mathbb{H}$, see, for instance, [46].

Theorem 8. Let $f: \mathbb{H} \rightarrow \mathbb{H}$ be a $C^{1}$-smooth orientation preserving contact map, and let $c_{\theta \alpha}(r)=f\left(\phi_{\theta \alpha}(r)\right)$. Set $1 / p+1 / q=1, p, q>1$, and

$$
\ell(\theta, \alpha)=\int_{1}^{R}\left(\frac{\left\|\dot{c}_{\theta \alpha}\right\|_{0}}{J_{f} r^{3} \sqrt{\cos \alpha}}\right)^{q} J_{f} r^{3} \sqrt{\cos \alpha} d r, \quad \alpha \in[-\pi / 2, \pi / 2], \quad \theta \in[0,2 \pi) .
$$

Then

$$
\rho_{0}(y)=\frac{1}{\ell(\theta, \alpha)}\left(\frac{\left\|\dot{c}_{\theta \alpha}\right\|_{0}}{J_{f} r^{3} \sqrt{\cos \alpha}}\right)^{\frac{1}{p-1}} \circ f^{-1}, \quad G(\theta, \alpha, r) \in R_{1 b},
$$

$y=f\left(x_{1}, x_{2}, t\right) \in R_{1 b}^{\prime}=f\left(R_{1 b}\right)$, is the extremal function for the $p$-module $M_{p}\left(f\left(\Gamma_{0}\right)\right)$ and moreover $M_{p}\left(f\left(\Gamma_{0}\right)\right)=\int_{R_{1 b}^{\prime}} \rho_{0}^{p} d y=\int_{-\pi / 2}^{\pi / 2} \int_{0}^{2 \pi} \ell^{1-p} d \theta d \alpha$.

Proof. Let us first observe that $\int_{c_{\theta \alpha}} \rho_{0} d s=1$, where $d s$ is the arc-length element defined with respect to the norm $N_{\mathbb{H}}$. Indeed,

$$
\begin{aligned}
\int_{c_{\theta \alpha}} \rho_{0} d s & =\int_{1}^{b}\left(\rho_{0} \circ f\right)\left\|\dot{c}_{\theta \alpha}\right\|_{0} d r=\frac{1}{\ell} \int_{1}^{b}\left(\frac{\left\|\dot{c}_{\theta \alpha}\right\|_{0}}{J_{f} r^{3} \sqrt{\cos \alpha}}\right)^{\frac{1}{p-1}}\left\|\dot{c}_{\theta \alpha}\right\| d r \\
& =\frac{1}{\ell} \int_{1}^{b}\left(\frac{\left\|\dot{c}_{\theta \alpha}\right\|_{0}}{J_{f} r^{3} \sqrt{\cos \alpha}}\right)^{q} J_{f} r^{3} \sqrt{\cos \alpha} d r=1
\end{aligned}
$$

for all $\alpha \in(-\pi / 2, \pi / 2)$ and $\theta \in[0,2 \pi)$. Therefore, $\rho_{0}$ is admissible for $f\left(\Gamma_{0}\right)$ and

$$
M_{p}\left(f\left(\Gamma_{0}\right)\right) \leq \int_{R_{1 b}^{\prime}} \rho_{0}^{p} d \mathbf{g}
$$


On the other hand, for any $\rho$ admissible for $f\left(\Gamma_{0}\right)$ we have $\int_{c_{\theta \alpha}} \rho d s \geq 1$, and therefore,

$$
\int_{c_{\theta \alpha}}\left(\rho-\rho_{0}\right) d s \geq 0
$$

This implies that

$$
\frac{1}{\ell^{p-1}(\theta, \alpha)} \int_{1}^{b}\left[\left(\rho-\rho_{0}\right) \circ f\right]\left\|\dot{c}_{\theta \alpha}\right\|_{0} d r \geq 0
$$

Then

$$
\int_{S_{1} \backslash \mathcal{Z}} \int_{1}^{b}\left(\left(\rho-\rho_{0}\right) \rho_{0}^{p-1} \circ f\right) J_{f} r^{3} d r d H^{3} \geq 0 .
$$

Equivalently,

$$
\int_{R_{1 b}^{\prime}} \rho \rho_{0}^{p-1} d \mathbf{g} \geq \int_{R_{1 b}^{\prime}} \rho_{0}^{p} d \mathbf{g}
$$

The Hölder inequality yields

$$
\left(\int_{R_{1 b}^{\prime}} \rho^{p} d \mathbf{g}\right)^{1 / p}\left(\int_{R_{1 b}^{\prime}} \rho_{0}^{(p-1) q} d \mathbf{g}\right)^{1 / q} \geq \int_{R_{1 b}^{\prime}} \rho \rho_{0}^{p-1} d \mathbf{g} \geq \int_{R_{1 b}^{\prime}} \rho_{0}^{p} d \mathbf{g},
$$

or since $(p-1) q=p$,

$$
\int_{R_{1 b}^{\prime}} \rho^{p} d \mathbf{g} \geq \int_{R_{1 b}^{\prime}} \rho_{0}^{p} d \mathbf{g}
$$

Taking infimum in the above inequality over all admissible $\rho$ we conclude that

$$
M_{p}\left(f\left(\Gamma_{0}\right)\right) \geq \int_{R_{1 b}^{\prime}} \rho_{0}^{p} d \mathbf{g}
$$

Comparing (35) and (36) we see that the function $\rho_{0}$ is extremal for the module $M_{p}\left(f\left(\Gamma_{0}\right)\right)$. Now we can calculate the $p$-module as

$$
\begin{aligned}
M_{p}\left(f\left(\Gamma_{0}\right)\right) & =\int_{R_{1 b}^{\prime}} \rho_{0}^{p} d \mathbf{g}=\int_{S^{1} \backslash \mathcal{Z}} \int_{1}^{b}\left[\rho_{0}^{p} \circ f\right] J_{f} r^{3} d r d H^{3} \\
& =\int_{S_{1} \backslash \mathcal{Z}} \int_{1}^{b} \frac{1}{\ell^{p}(\theta, \alpha)}\left(\frac{\left\|\dot{c}_{\theta \alpha}\right\|_{0}}{J_{f} r^{3} \sqrt{\cos \alpha}}\right)^{\frac{p}{p-1}} J_{f} r^{3} d r d H^{3} \\
& =\int_{-\pi / 2}^{\pi / 2} \int_{0}^{2 \pi} \ell^{1-p}(\theta, \alpha) d \theta d \alpha
\end{aligned}
$$

Example 6. The $p$-module of $\Gamma=\Gamma\left(R_{a, b} ; S_{a}, S_{b}\right)$

$$
M_{p}\left(\Gamma_{0}\right)=M_{p}\left(\Gamma_{0}\right)= \begin{cases}\frac{2 \pi \sqrt{\pi} \Gamma\left(\frac{p}{4}+\frac{1}{2}\right)}{\Gamma\left(\frac{p}{4}+1\right)}\left(\frac{p-4}{p-1}\right)^{p-1}\left(b^{\frac{p-4}{p-1}}-1\right)^{1-p}, & \text { for } p \neq 4 \\ \frac{\pi^{2}}{(\log b)^{3}}, & \text { for } p=4\end{cases}
$$


was calculated in [47].

Let us now calculate the $p$-module of $f\left(\Gamma_{0}\right)$, where $f$ is a contact $C^{1}$-smooth orientation preserving map. If we try to create a twisting map similarly to Example 5 in the spherical coordinates written as

$$
G^{-1} \circ f \circ G:(\theta, \alpha, r) \rightarrow(\theta+\omega(r), \alpha, r), \quad \omega(1)=0,
$$

with $G$ defined by (34), (i.e, the boundary sphere $S_{1}$ remains unchanged while the spheres $S_{r}$ rotate to the angle $\left.\omega(r), r \in(1, b]\right)$, then the condition of horizontality for the curves $f\left(\phi_{\theta \alpha}\right)$ is quite rigid, which leads us to $\omega(r) \equiv 0$. Let us try to modify the twisting map by

$G^{-1} \circ f \circ G:(\theta, \alpha, r) \rightarrow\left(\theta+\tan \alpha \log r+\omega_{1}(r), \alpha+\omega_{2}(r), r\right), \quad \omega_{1}(1)=\omega_{2}(1)=0$.

Then the image $c_{\theta \alpha}(r)=f\left(\phi_{\theta \alpha}(r)\right)$ is written in coordinates as

$$
\begin{aligned}
x_{1}(r) & =r \sqrt{\left|\cos \left(\alpha+\omega_{2}(r)\right)\right|} \cos \left(\theta+\omega_{1}(r)\right), \\
x_{2}(r) & =r \sqrt{\left|\cos \left(\alpha+\omega_{2}(r)\right)\right|} \sin \left(\theta+\omega_{1}(r)\right), \\
t(r) & =r^{2} \sin \left(\alpha+\omega_{2}(r)\right) .
\end{aligned}
$$

The horizontality condition $\dot{t}=2\left(\dot{x}_{1} x_{2}-\dot{x}_{2} x_{1}\right)$ is equivalent to

$$
\dot{\omega}_{1}=-\frac{1}{2} \dot{\omega}_{2}-\frac{1}{r} \tan \left(\alpha+\omega_{2}\right) \text {. }
$$

For example,

$$
\omega_{2}(r)=r-1, \quad \omega_{1}=\frac{1-r}{2}-\int_{1}^{r} \frac{\tan (\alpha+s-1)}{s} d s .
$$

Then $J_{f}=J_{G^{-1} \circ f \circ G}=1$ and

$$
\left\|\dot{c}_{\theta \alpha}\right\|_{0}=\frac{1}{2} \sqrt{\frac{4+r^{2}}{\cos (\alpha+r-1)}} .
$$

In Theorem 8 we calculate

$$
\ell(\theta, \alpha)=\ell(\alpha)=\int_{1}^{b}\left(\frac{4+r^{2}}{4 \cos (\alpha+r-1)}\right)^{q / 2} r^{3-q}(\cos \alpha)^{\frac{1}{2}(1-q)} d r .
$$

and the $p$-module of $f\left(\Gamma_{0}\right)$ is

$$
M_{p}\left(f\left(\Gamma_{0}\right)\right)=2 \pi \int_{-\pi / 2}^{\pi / 2} \ell^{1-p}(\alpha) d \alpha .
$$

Unfortunately, the integral inequality which follows from the monotonicity of the module $M_{p}\left(f\left(\Gamma_{0}\right)\right) \leq M_{p}\left(\Gamma_{0}\right)$ is quite difficult, and there is very little hope to obtain simple inequalities as in Example 5. 


\section{REFERENCES}

[1] L. Ahlfors, Conformal Invariants: Topics in Geometric Function Theory, McGraw-Hill Book Co., New York, 1973.

[2] L. Ahlfors, Lectures on quasiconformal mappings, 2-nd. ed. University Lecture Ser., vol. 38, Amer. Math. Soc., 2006.

[3] L. Ahlfors and A. Beurling, Conformal invariants and function-theoretic null-sets, Acta Math., 83 (1950), no. 1-2, 101-129.

[4] G. D. Anderson, S. L. Qiu, M. K. Vamanamurthy, and M. Vuorinen, Generalized elliptic integrals and modular equations, Pac. J. Math. 192 (2000), no. 1-37.

[5] G. Anzellotti and R. Serapioni, $C^{k}$-rectifiable sets, J. Reine Angew. Math. 453 (1994), 1-20.

[6] H. Aikawa and M. Ohtsuka, Extremal length of vector measures, Ann. Acad. Sci. Fenn. Math. 24 (1999), no. 1, 61-88.

[7] Z. M. Balogh and J. T. Tyson, Polar coordinates in Carnot groups, Math. Z., 241, (2002), no. 4, 697-730.

[8] M. Badger, Beurling's criterion and extremal metrics for Fuglede modulus, Ann. Acad. Sci. Fenn., Math. 38 (2013), 677-689.

[9] B. Bojarski, P. Hajłasz, and P. Strzelecki, Sards theorem for mappings in Hölder and Sobolev spaces, Manuscripta Math. 118 (2005), 383-397.

[10] M. Brakalova, On the asymptotic behavior of some conformal and quasiconformal mappings, PhD Thesis, Sofia University, Sofia (1988), 1-115.

[11] M. A. Brakalova and J. A. Jenkins, On solutions of the Beltrami equation, J. Anal. Math. 76, (1998), 67-92.

[12] M. Brakalova and J. A. Jenkins, On a paper of Carleson, Ann. Acad. Sci. Fenn 27, (2002), 485-490.

[13] M. Brakalova, Sufficient and necessary conditions for conformality. Part II. Analytic viewpoint, Ann. Acad. Sci. Fenn. 35 (2010), 235-254.

[14] M. Brakalova, On Local stability of solutions to the Beltrami equation with degeneration, Compl. Anal.and Appl.'13, Proc. of International Conference, Sofia, 2013, (2013), 67-77.

[15] C. A. Cazacu, On the length-area dilatation, Complex. Var. Theory. Appl. 50 (2005), $1-21$.

[16] O. Calin, D. C. Chang, and P. Greiner, Geometric analysis on the Heisenberg group and its generalizations, AMS/IP Studies in Advanced Mathematics, 40. American Mathematical Society, Providence, RI; International Press, Somerville, MA, 2007. 244 pp

[17] L. Capogna, D. Danielli, and N. Garofalo, Capacitary estimates and the local behavior of solutions of nonlinear subelliptic equations, Amer. J. Math., 118, (1996), no. 6, 11531196.

[18] D. C. Chang and I. Markina, Anisotropic quaternion Carnot groups: geometric analysis and Green function, Adv. in Appl. Math. 39 (2007), no. 3, 345-394.

[19] M. Csörnyei, S. Hencl, and Y. Malý, Homeomorphisms in the Sobolev space $W^{1, n-1}$, J. Reine Angew. Math. 644 (2010), 221-235.

[20] V. Dubinin and M. Vuorinen, On conformal moduli of polygonal quadrilaterals. Israel J. Math, 171(1) (2007), 111-125.

[21] A. Ya. Dubovickiu,, On the structure of level sets of differentiable mappings of an $n$ dimensional cube into a k-dimensional cube, Izv. Akad. Nauk SSSR. Ser. Mat. 21 (1957), 371-408.

[22] H. Federer, Geometric measure theory, Die Grundlehren der mathematischen Wissenschaften, Band 153 Springer-Verlag New York Inc., New York 1969, 676 pp. 
[23] G. B. Folland and E. M. Stein, Hardy spaces on homogeneous groups, Mathematical Notes, 28, Princeton University Press, Princeton, N.J., 1982, 285 pp.

[24] G. B. Folland, Subelliptic estimates and function spaces on nilpotent Lie groups, Ark. Mat., 13, (1975), no. 2, 161-207.

[25] B. Fuglede, Extremal length and functional completion, Acta Math. 98 (1957), 171-219.

[26] J. Garnett and D. Marshall, Harmonic Measure, Cambridge University Press, New York (2005), 571 pp.

[27] F. W. Gehring, Symmetrizaition of rings in space, Trans. Amer. Math. Soc. 101 (1961), 499-519.

[28] F. W. Gehring, Rings and quasiconformal mappings in space, Trans. Amer. Math. Soc. 103 (1962), 353-393.

[29] F. W. Gehring, Extremal length definitions for the conformal capacity of rings in space, Michigan Math. J. 9 (1962), 137-150.

[30] V .M. Goldshtein and A. S. Romanov, Transformations that preserve Sobolev spaces, Sibirsk. Mat. Zh. 25 (1984), no. 3, 55-61; English transl. in Siberian Math. J. 25 (1984), no. $3,382-388$.

[31] H. Grötzsch, Eleven papers, Ber. Verh. Sächs. Acad. Wiss., Leipzig, Math. Phys. (19281932).

[32] V. Gutlyanskii and O. Martio, Conformality of a quasiconformal mapping at a point, J. Anal. Math. 91 (2003), 179-192.

[33] P. Hajłasz, Change of variables formula under minimal assumptions, Colloquium Mathematicum LXVI 1993, 93-101.

[34] P. Hajłasz, Sobolev mappings, co-area formula and related topics, In: Proceedings on Analysis and Geometry (Novosibirsk Akademgorodok, 1999), Izdat. Ross. Akad. Nauk Sib. Otd. Inst. Mat., Novosibirsk, 2000, 227-254.

[35] H. Hakula, A. Rasila, and M. Vuorinen, On moduli of rings and quadrilaterals: algorithms and experiments, SIAM J. Sci. Comput. 33, (2011), 279-302.

[36] J. Heinonen, Calculus on Carnot groups, Fall School in Analysis, Juväskylä 1994. Ber. Univ. Juväskylä Math. Inst. 68 (1995), 1-31.

[37] J. Heinonen and I. Holopainen, Quasiregular maps on Carnot groups, J. Geom. Anal. 7 (1997), no. 1, 109-148.

[38] S. Hencl and J. Malý, Jacobians of Sobolev homeomorphisms, Calc. Var. 38 (2010), 233-242.

[39] J. Hesse, A p-extremal length and p-capacity equality, Ark. Mat. 13 (1975), 131-144.

[40] T. Iwaniec and G. Martin, Geometric function theory and non-linear analysis, Oxford Mathematical Monographs. The Clarendon Press, Oxford University Press, New York, 2001.

[41] J. A. Jenkins, Univalent functions and conformal mapping, Ergebnisse der Mathematik und ihre Grenzgebiete, Springer, Berlin-Göttingen-Heidelberg, 1958, 169 pp.

[42] H. Hakula, A. Rasila, and M. Vuorinen On moduli of rings and quadrilaterals: algorithms and experiments, SIAM J. Sci. Comput. 33 (2011), 279302.

[43] A. Kaplan, Fundamental solutions for a class of hypoelliptic PDE generated by composition of quadratic forms, Trans. Amer. Math. Soc. 258 (1980), 147-153.

[44] M. Karmanova and S. Vodop'yanov, Carnot-Carathéodory spaces, coarea and area formulas, Analysis and Mathematical Physics, Trends in Mathematics, Birkhäuser 2009, 233-335.

[45] A. Korányi, Poisson formulas for circular functions on some groups of type $H$, Sci. China. Ser. A: Math. 49 (2006), no. 11, 1683-1695. 
[46] A. Korányi and H. M. Reimann, Quasiconformal mappings on the Heisenberg group, Invent. Math. 80 (1985), no. 2, 309-338.

[47] A. Korányi and H. M. Reimann, Horizontal normal vectors and conformal capacity of spherical rings in the Heisenberg group, Bull. Sci. Math. (2) 111 (1987), no. 1, 3-21.

[48] V. V. Krivov, Some properties of moduli in space, Dokl. Akad. Nauk, SSSR, 154 (1964), $510-513$.

[49] O. Lehto, Homeomorphisms with a given dilatation, Lecture Notes in Math. 118, (1969), $58-73$.

[50] O. Lehto and K. I. Virtanen, Quasiconformal Mappings in the Plane, Springer Verlag, 1973.

[51] C. Löwener, On the conformal capacity in space, J. Math. Mech. 8 (1959), 411-414.

[52] V. Magnani, The coarea formula for real-valued Lipschitz maps on stratified groups, Math. Nachr. 278 (2005), no. 14, 1689-1705.

[53] J. Malý, D. Swanson, and W. P. Ziemer, The co-area formula for Sobolev mappings, Trans. Amer. Math. Soc. 355 (2003), no. 2, 477-492.

[54] I. Markina, On coincidence of p-module of a family of curves and p-capacity on the Carnot group, Rev. Mat. Iberoamericana, 19, (2003), no. 1, 143-160.

[55] I. Markina, Extremal widths on homogeneous groups. Complex Var. Theory Appl. 48 (2003), no. 11, 947-960.

[56] I. Markina, p-module of vector measures in domains with intrinsic metric on Carnot groups, Tohoku Math. J. (2) 56 (2004), no. 4, 553-569.

[57] I. Markina, Modules of vector measures on the Heisenberg group, Complex analysis and dynamical systems II, 291304, Contemp. Math., 382, Amer. Math. Soc., Providence, RI, 2005.

[58] M. Marcus and V. J. Mizel, Complete characterization of functions which act, via superposition, on Sobolev spaces, Trans. Amer. Math. Soc. 251 (1979), 187-218.

[59] J. Mitchell, On Carnot-Carathodory metrics, J. Differential Geom. 21 (1985), no. 1, $35-45$.

[60] F. Morgan, Geometric measure theory, a beginner's guide, 2nd ed., Academic Press, Boston, 1995, 249 pp.

[61] M. Ohtsuka, Extremal length and precise functions. With a preface by Fumi-Yuki Maeda, GAKUTO International Series. Mathematical Sciences and Applications, 19. Gakkōtosho Co., Ltd., Tokyo, 2003, 343 pp.

[62] P. Pansu, Métriques de Carnot-Carathéodory et quasiisométries des espaces symétriques de rang un, (French) [Carnot-Carathéodory metrics and quasi-isometries of rank-one symmetric spaces] Ann. of Math. (2) 129 (1989), no. 1, 1-60.

[63] C. Pommeremke, Boundary behavior of conformal maps, Springer Verlag, Berlin Heidelberg, 1992, $300 \mathrm{pp}$.

[64] A. Rasila and M. Vuorinen, Experiments with moduli of quadrilaterals, Rev. Roumaine Math. Pures Appl. 51 (2006), no. 5-6, 747-757.

[65] E. Reich, Steiner symmetrization and the conformal Moduli of parallelograms, Analysis and Topology, a volume dedicated to the memory of Stoilow. (1998), 615-620.

[66] E. Reich and H. Walczak, On the behavior of quasiconformal mappings at a point, Trans. Amer. Math. Soc. 117 (1965), 338-351.

[67] Y. G. Reshetnyak, The concept of capacity in the theory of functions with generalized derivatives, Siberian Math. J. 10 (1969), no. 5, 818-842.

[68] H. M. Riemann, H-type groups and Clifford modules, Adv. Appl. Clifford Alg. 11 (2001), no. S2, 277-288. 
[69] B. Rodin, The method of extremal length, Bull. Amer. Math. Soc. 80 (1974), no. 4, 587-606.

[70] B. Rodin and S. E. Warschawski, Extremal length and the boundary behavior of conformal mappings, Ann. Acad. Sci. Fenn. Ser. A I Math. 2 (1976), 467-500.

[71] B. Rodin and S. E. Warschawski, Extremal length and univalent functions. I. The angular derivative, Math. Z. 153 (1977), no. 1, 1-17.

[72] J. Serrin, Local behaviour of solution of quasi-linear equation, Acta Math. 111 (1964), 247-302.

[73] N. Shanmugalingam, Newtonian spaces: an extension of Sobolev spaces to metric measure spaces, Rev. Mat. Iberoamericana 16 (2000), no. 2, 243-279.

[74] V. A. Shlyk, Capacity of a condenser and the modulus of a family of separating surfaces, (Russian) Zap. Nauchn. Sem. Leningrad. Otdel. Mat. Inst. Steklov. (LOMI) 185 (1990), Anal. Teor. Chisel i Teor. Funktsii. 10, 168-182, 187; translation in J. Soviet Math. 59 (1992), no. 6, 1240-1248

[75] V. A. Shlyk, On the equality between p-capacity and p-modulus, (Russian) Sibirsk. Mat. Zh. 34 (1993), no. 6, 216-221; translation in Siberian Math. J. 34 (1993), no. 6, 11961200 .

[76] B. V. Šabat, The modulus method in space, Dokl. Akad. Nauk SSSR 130 1210-1213 (Russian); translated as Soviet Math. Dokl. 1 (1960), 165-168.

[77] O. Teichmüller, Untersuchungen über konforme und qusiconforme Abbildung, Deutsche Math. 3 (1938), 621-678.

[78] A. Vasil'ev, Moduli of families of curves for conformal and quasiconformal mappings, Lecture Notes in Mathematics, 1788. Springer-Verlag, Berlin, 2002, 211 pp.

[79] S. K. Vodopyanov and V. M. Goldshtein, Functional characteristics of quasiisometric mappings, Sibirsk. Mat. Zh. 17 (1976), no. 4, 768-773; English transl. in Siberian Math. J. 17 (1976), no. 4, 580-584.

[80] S. K. Vodopyanov, Mappings of homogeneous groups and imbeddings of functional spaces, Sibirsk. Mat. Zh. 30 (1989), no. 5, 25-41; English transl. in Siberian Math. J. 30 (1989), no. 5, 685-698.

[81] S. K. Vodopyanov, Potential theory on homogeneous groups, Mat. Sb. 180 (1989), no. 1, 57-77; English transl. in Math. USSR-Sb. 66 (1990), no. 1, 59-81.

[82] S. K. Vodopyanov and A. D. Ukhlov, Superposition operators in Sobolev spaces, Izv. Vyssh. Uchebn. Zaved. Mat. (2002), no. 10, 11-33; tEnglish transl. in Russian Math. (Iz. VUZ) 46 (2002), no. 10, 9-31.

[83] S. K. Vodopyanov, Composition operators on Sobolev spaces, In: Complex Analysis and Dynamical Systems II: A Conference in Honor of Professor Lawrence Zalcman's $60^{\text {th }}$ birthday, June 9-12, 2003, Nahariya, Israel. Contemporary Math., vol. 382, 2005, pp. $327-2342$.

[84] S. K. Vodopyanov, Regularity of mappings inverse to Sobolev mappings, Mat. Sb. 203 (2012), no. 10, 3-32; English transl. Sbornik. Math. 203 (2012), no. 10, 1383-1410.

[85] J. Väisälä, On quasiconformal mapping in space, Ann. Acad. Sci. Fenn. Ser. AI 296, (1961), 1-36.

[86] J. Väisälä, Lectures on n-dimensional quasiconformal mappings, Lecture Notes in Math. 229, Springer-Verlag, Berlin-New York, 1971.

[87] L. I. Volkovyskii, Investigation of the type problem for a simply connected Riemann surface, Trudy Mat. Inst. Steklov., Acad. Sci. USSR, MoscowLeningrad, 34, (1950) 3171. 
[88] W. P. Zimmer, Extremal length and conformal capacity, Trans. Amer. Math. Soc. 126 (1967), 460-473.

[89] W. P. Zimmer, Extremal length and p-capacity, Michigan Math. J. 16 (1969), 43-51.

[90] W. P. Zimmer, Extremal length as a capacity, Michigan Math. J. 17 (1970), 117-128.

M. Brakalova: Department of Mathematics, Fordham University, 407 John Mulcahy Hall Bronx, NY 10458-5165, USA

E-mail address: brakalova@fordham.edu

I. Markina and A. Vasil'ev: Department of Mathematics, University of Bergen, P.O. Box 7800, Bergen N-5020, Norway

E-mail address: irina.markina@uib.no

E-mail address: alexander.vasiliev@math.uib.no 